Cite this: Phys. Chem. Chem. Phys., $2014,16,3442$

Received 4th September 2013, Accepted 16th December 2013 DOI: $10.1039 / c 3 c p 53767 f$

www.rsc.org/pccp

\section{The structure and dynamics of carbon dioxide and water containing ices investigated via $\mathrm{THz}$ and mid-IR spectroscopy}

\author{
Marco A. Allodi, ${ }^{a}$ Sergio loppolo, ${ }^{\text {bc }}$ Matthew J. Kelley, $\dagger^{a}$ Brett A. McGuire ${ }^{a}$ and \\ Geoffrey A. Blake*ab
}

\begin{abstract}
Icy dust grains play a key role in the chemistry of the interstellar medium. The cumulative outcome of recent observations, laboratory studies, and astrochemical models indicates that solid-phase reaction mechanisms may dominate the formation of complex organic molecules such as amino acids and sugars in space. Consequently, the composition and structure of the icy grain mantle may significantly influence solid-phase reaction pathways. In this work, we present a new experimental setup capable of studying astrochemical ice analogs in both the TeraHertz $(\mathrm{THz})$, or far-Infrared (far-IR), region $(0.3-7.5 \mathrm{THz}$; $\left.10-250 \mathrm{~cm}^{-1}\right)$ and the mid-IR $\left(400-4000 \mathrm{~cm}^{-1}\right)$. The instruments are capable of performing a variety of spectroscopic studies that can provide especially relevant laboratory data to support astronomical observations from telescopes such as Herschel, SOFIA, and ALMA. Experimental spectra of astrochemical ice analogs of water and carbon dioxide in pure, mixed, and layered ices were collected at different temperatures under high vacuum conditions with the goal of investigating the structure of the ice. We tentatively observe a new feature in both amorphous solid water and crystalline water at $33 \mathrm{~cm}^{-1}$ $(1 \mathrm{THz})$. In addition, our studies of mixed and layered ices show how it is possible to identify the location of carbon dioxide as it segregates within the ice by observing its effect on the $\mathrm{THz}$ spectrum of water ice. The THz spectra of mixed and layered ices are further analyzed by fitting their spectral features to those of pure amorphous solid water and crystalline water ice to quantify the effects of temperature changes on structure. From the results of this work, it appears that $\mathrm{THz}$ spectroscopy is potentially well suited to study thermal transformations within the ice.
\end{abstract}

\section{Introduction}

Dust grains play a central role in the chemistry of the interstellar medium (ISM). Hydrogen atoms only react efficiently to form molecular hydrogen with a dust grain acting as a third body to stabilize the new molecular species. ${ }^{1-4}$ In low-mass star-forming regions, analogous to the cloud that formed our own solar system, there exist cores with low enough local temperatures, and high enough density for gas to condense onto dust grains. Simple, gasphase atoms and molecules accrete onto a dust grain to form an icy outer mantle, and new reaction pathways become available. ${ }^{5-7}$

\footnotetext{
${ }^{a}$ Division of Chemistry and Chemical Engineering, California Institute of Technology, 1200 E California Blvd., Pasadena, CA 91125, USA. E-mail: mallodi@caltech.edu,gab@gps.caltech.edu

${ }^{b}$ Division of Geological and Planetary Science, California Institute of Technology, 1200 E California Blvd., Pasadena, CA 91125, USA

${ }^{c}$ Faculty of Science, Radboud University Nijmegen, IMM, P.O. Box 9010, NL 6500 GL Nijmegen, The Netherlands

$\dagger$ Present Address: Technology and Applications Center, Newport Corporation, 1791 Deere Avenue, Irvine, CA 92606, USA.
}

The diversity of chemistry possible on the ice surface greatly exceeds the possibilities in the gas phase. In an ice, energetic processing, by UV photons or cosmic rays, excites or breaks chemical bonds, leading to chemical reactions of species within the ice. ${ }^{8-13}$ Thermal processing can occur if different molecules present in the ice initially do not have enough energy to react, and then later gain sufficient energy to overcome a reaction barrier. ${ }^{14-16}$ In addition, atoms and light molecules freeze out onto the grains and diffuse rapidly over the ice surface inducing chemical reactions. ${ }^{17-24}$ The ice facilitates the interaction between different species and also serves as a third body to stabilize a new molecule if it forms.

Consequently, these solid-phase reaction mechanisms can potentially lead to the formation of more complex molecules such as sugars and amino acids. Given that recent work has ruled out a gas-phase mechanism in glycine formation, ${ }^{25}$ and yet glycine has been detected in comets sampled by the STARDUST mission, ${ }^{26}$ solid-phase reaction mechanisms on ice grains may provide the main pathway for the formation of complex organic molecules (COMs) in the ISM. ${ }^{27}$ In addition, molecular ices on 
dust grains likely play a fundamental role in the chemistry of planetary ices, which have been observed on different bodies within our solar system. ${ }^{28,29}$

The role that the structure of the ice mantle plays in chemical reactions on icy grain surfaces remains an incompletely explored question in understanding the effect of dust grains on the chemistry of the ISM. Given the icy dust grains' ability to facilitate the interaction between different species within the ice as discussed above, it would be useful to understand, specifically, how the ice structure can impact diffusion barriers within the ice. Since COMs form as smaller molecules diffuse through an ice, accurate models of diffusion barriers will improve COM formation models. ${ }^{27,30}$

Mid-infrared (mid-IR) spectroscopy (450-4000 $\mathrm{cm}^{-1}$ ) has been fundamental in improving our understanding of ice and dust in the ISM. ${ }^{31-33}$ As a result, the most abundant simple molecules have been detected in the solid phase via mid-IR astronomical observations. ${ }^{34}$ To begin investigating structural effects in ices, many laboratory investigations have been carried out on ice mixtures composed of $\mathrm{H}_{2} \mathrm{O}$ and $\mathrm{CO}_{2}$, the principal focus of this work. Early studies by Bernstein et al. reported how the intermolecular interactions can break the symmetry of different molecules, and thus cause significant changes in the IR spectra, including the presence of peaks not observed in a pure $\mathrm{CO}_{2}$ ice. ${ }^{35}$ Galvez et al. and Maté et al. characterized extensively co-deposited and sequentially-deposited $\mathrm{H}_{2} \mathrm{O}$ and $\mathrm{CO}_{2}$ ice. ${ }^{36-38}$ Their work detected structural differences between mixed ices and identified the differences between $\mathrm{CO}_{2}$ molecules that formed a crystalline layer on top of $\mathrm{H}_{2} \mathrm{O}$, and the $\mathrm{CO}_{2}$ molecules that diffused into the $\mathrm{H}_{2} \mathrm{O}$ sample using Fouriertransform IR (FTIR) spectroscopy and temperature-programmed desorption (TPD) experiments. Öberg et al. investigated segregation within mixed $\mathrm{CO}_{2}$ and $\mathrm{H}_{2} \mathrm{O}$ ice and found that molecules will form segregated groups as long as it remains more favorable to interact with like molecules as opposed to different species. ${ }^{39}$ In addition, they also showed that, in contrast with what was previously thought, ice dynamics were thickness dependent. Clearly, IR spectroscopy offers valuable insight into the structure of the ice. However, transitions at IR frequencies correspond to intramolecular vibrational modes of the molecules in the ice, and, thus, IR spectroscopy only detects local changes in structure.

In contrast, TeraHertz (THz), or far-IR, spectroscopy, covering the region from 0.1 to $10 \mathrm{THz}\left(3-330 \mathrm{~cm}^{-1}\right)$, directly probes lower frequency vibrations that correspond to low energy intraand, especially, intermolecular modes. By virtue of probing intermolecular modes, $\mathrm{THz}$ spectroscopy is particularly sensitive to the long range interactions between molecules. ${ }^{40}$ Since any change to the structure of the ice would affect these long range interactions, $\mathrm{THz}$ spectroscopy allows for direct measurements of large-scale structural change. While there has been previous spectroscopic work done in this region, ${ }^{4-45}$ none of the previous experiments focused on understanding the structure of mixed and layered ices.

There has been some theoretical work focused on mixed and layered $\mathrm{H}_{2} \mathrm{O}$ and $\mathrm{CO}_{2}$ ices. ${ }^{46,47}$ Both works focus on the suitability of the mid- and far-IR for studying structural changes in astrophysically-relevant ices. Lee et al. find a wealth of spectroscopic features in the $\mathrm{THz}$ region and conclude that the features of $\mathrm{H}_{2} \mathrm{O}$ ice dominate the spectrum in this region. In addition, they find ice layers at different distances from an $\mathrm{H}_{2} \mathrm{O}-\mathrm{CO}_{2}$ interface can have different spectral features which are particularly well pronounced at $\mathrm{THz}$ wavelengths. ${ }^{47}$ Their results encourage work in the $\mathrm{THz}$ region to investigate these effects experimentally. As observational facilities such as Herschel, ${ }^{48}$ SOFIA, ${ }^{49}$ and ALMA $^{50}$ continue to produce high-quality sub-millimeter and $\mathrm{THz}$ data, complementary laboratory data can provide important structural information about the ice and, indeed, give the scientific community the tools needed to interpret observational spectra and understand ice chemistry in the ISM and exoplanetary systems.

In the present study, we employ $\mathrm{THz}$ and mid-IR spectroscopy to characterize astrochemical ice analogs of $\mathrm{H}_{2} \mathrm{O}$ and $\mathrm{CO}_{2}$. We first describe our state-of-the-art setup, which includes a detailed discussion of the time-domain (TD) THz spectrometer built in our lab. To investigate the ice structure, we prepared ices of pure $\mathrm{H}_{2} \mathrm{O}$ or $\mathrm{CO}_{2}$. We then prepared ices composed of distinct molecular layers, as well as mixtures that were co-deposited, and acquired THz and mid-IR spectra after ice deposition and at different temperatures. Our results provide insight into how different layers of ice interact with each other, and how the segregation of more volatile molecules within an ice can affect the its short- and long-range structure, thus demonstrating the importance of $\mathrm{THz}$ spectroscopy in astrochemical ice studies. These laboratory investigations have potential implications for future astronomical work.

\section{Experimental methods}

The new experimental setup used in this work consists of a high vacuum chamber; a commercially available FTIR spectrometer to cover the mid-IR; and a TD THz spectrometer, constructed in-house, to cover the $\mathrm{THz}$, or far-IR, region. The complete instrument can be seen in Fig. 1. We will discuss each component in detail in the sections below.

\subsection{Vacuum chamber and IR spectrometer}

Studying astrochemically-relevant ice analogues requires high vacuum and low temperatures. To achieve these conditions, we employ a high vacuum chamber (R. C. Janis) that encloses the cold finger of a closed-cycle $\mathrm{He}$ cryostat (CTI Cryogenics) capable of cooling a $1^{\prime \prime}$, high-resistivity, intrinsic Si substrate (University Wafers) to $\sim 8 \mathrm{~K}$. A heating element, and thermocouple connected to the cryostat allows for precise control of the substrate temperature between $\sim 8 \mathrm{~K}$ and $300 \mathrm{~K}$. As seen in Fig. 2, the vacuum chamber is pumped through a $1.5^{\prime \prime}$ diameter, flexible, stainless steel pipe connected to a turbomolecular pump (Turbo V 250 Varian inc.). The configuration allows for the facile vertical translation of the main vacuum chamber when necessary to align the spectrometers. A liquid nitrogen reservoir above the TMP can be filled for cryopumping. The room temperature base pressure in the vacuum chamber reaches $\sim 5 \times 10^{-6}$ Torr, yet when the cryostat 


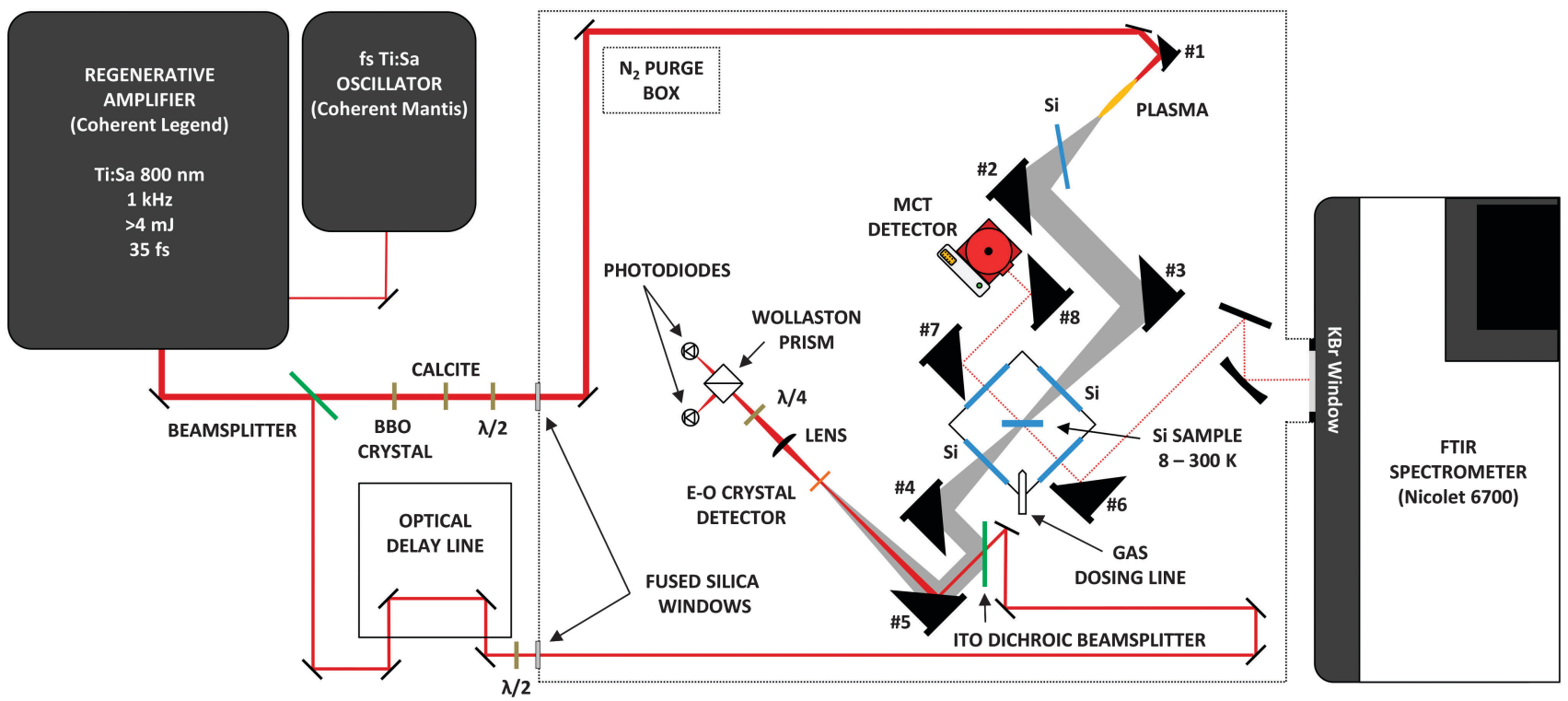

Fig. 1 The layout of the Caltech astrochemical ice analog experimental setup, including the home-built time-domain THz spectrometer (not to scale). The mirrors used to steer the generation beam before the BBO are low dispersion, dielectric, high-reflecting mirrors (Newport 10B20UF.20). The 400 and $800 \mathrm{~nm}$ beams for generation enter the purge box via a dual-band AR coated 1" thick fused silica window (Eksma). The $800 \mathrm{~nm}$ detection beam enters the purgebox via $800 \mathrm{~nm}$ AR coated 1" thick fused silica window (Eksma). The mirrors used to steer the detection beam are protected silver mirrors (Thorlabs PF10-03-P01). Two beam samplers (Newport 10B20-01NC.2) are used in place of 2 mirrors along the detection path to attenuate the beam power, so as not to saturate the photodiodes. The gold coated, $1^{\prime \prime}$ diameter, $8^{\prime \prime}$ focal length ( $f$ ) OAPM (Edmunds) labeled \#1 above is used to generate the air plasma. OAPMs \#2-4 used to steer the THz beam are $2^{\prime \prime}$ diameter protected gold mirrors with $f=6^{\prime \prime}$ (Edmunds Optics). To ensure a tight focus onto the crystal, OAPM \#5 has a $2^{\prime \prime}$ focal length. The OAPM \#6 $\left(f=4^{\prime \prime}\right)$ steers the mid-IR beam onto the sample, and the beam is collected by OAPM \#7 $\left(f=4^{\prime \prime}\right)$, and finally, tightly focused by OAPM \#8 $\left(f=2^{\prime \prime}\right)$ onto the MCT detector.

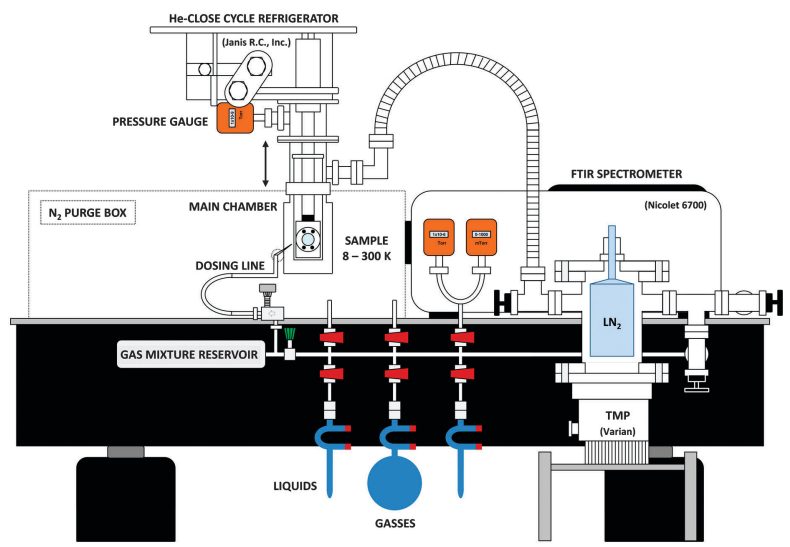

Fig. 2 A side view of the experimental setup. The THz spectrometer is not shown here for purposes of clarity.

is operating, the pressure in the vacuum chamber reaches as low as $\sim 10^{-8}$ Torr.

Samples are prepared for deposition in a stainless-steel dosing line as seen at the bottom of Fig. 2. The line implements a Pirani gauge (Lesker KJL275800) in the range of $10^{-4}$ to 1000 Torr, and an active capacitance transmitter (MKS Baratron) in the range between 0.01 to 1100 Torr. The latter allows for mass independent monitoring of the pressure of the gas sample in the line. An all-metal leak valve (Lesker) separates the chamber from the portion of the dosing line where samples enter or are mixed. A $1 / 8^{\prime \prime}$ diameter stainless steel pipe connects the leak valve to the vacuum chamber. The end of the pipe is capped with a metal mesh with a $38 \mu \mathrm{m}$ hole size (McMaster-Carr 85385T117) to ensure a uniform ice deposition, and the end is positioned $\sim 1^{\prime \prime}$ away from the substrate.

To deposit a pure ice, vapor fills the evacuated dosing line to a fixed pressure. Then, the leak valve leading into the chamber is opened to allow for a slow deposition of ice onto the $\mathrm{Si}$ substrate. The leak valve is closed once the ice on the substrate reaches the desired thickness. To create a layered sample, the dosing line is evacuated and filled with another molecule to a fixed pressure and then deposited via the leak valve.

Sample mixtures are prepared by first filling the dosing line with a sample of one molecule and then closing the valve separating the gas mixture reservoir from the rest of the dosing line. The remaining molecules of the first sample are evacuated using a mechanical pump and the dosing line is refilled with the second molecule. After filling the dosing line with the second molecule, the valve closing the gas mixture reservoir is opened and the two gasses mix in the full volume of the dosing line. The mixture can then be deposited onto the substrate in the sample chamber by opening the leak valve. For the experiments discussed below, water vapor was prepared in the dosing line from a sample of deionized liquid $\mathrm{H}_{2} \mathrm{O}$, which was degassed by several freeze-pump-thaw cycles. Gaseous $\mathrm{CO}_{2}$ was used as received from Air Liquide (99.99\%).

Our spectrometer operates in two distinct regions of the electromagnetic spectrum. Therefore, two different sources of radiation are used, each entering the chamber along separate 
paths but at $45^{\circ}$ angles to the substrate surface to allow for both beams to be incident on the same sample, as seen in Fig. 1. High-resistivity, $3.5 \mathrm{~mm}$ thick, $2^{\prime \prime}$ diameter, intrinsic Si wafers (University Wafers) act as both windows along the $\mathrm{THz}$ path, and one of the two windows along the IR path. A 2 " diameter Si wafer (Edmunds \#68-532) serves as the second IR window.

Mid-IR spectra with a resolution of $1 \mathrm{~cm}^{-1}$ are collected with a Nicolet 6700 FTIR spectrometer using OMNIC software (version 7.4.127, Thermo Scientific). Running in external sample mode, light leaves the spectrometer through a $2^{\prime \prime}$ diameter $\mathrm{KBr}$ window and enters a box purged with $\mathrm{N}_{2}$. A pair of spherical mirrors collimates the light out of the spectrometer, then an off-axis parabolic mirror (OAPM, Edmund Optics) focuses the light onto the sample to a spot size of $\sim 3 / 4^{\prime \prime}$ in diameter. OAPMs collect the light transmitted by the sample and steer the beam onto a mercury cadmium telluride (MCT) detector (Thermo Scientific 840-070300). All of the mid-IR spectra of ice samples shown below are averages of 512 co-added scans.

\subsection{THz spectrometer}

As seen in Fig. 1, the Caltech TD THz spectrometer relies upon an ultrafast laser system to generate and detect $\mathrm{THz}$ pulses. A Ti:Sapphire oscillator (Coherent Mantis) delivers sub 100 fs duration seed pulses containing $80 \mathrm{~nm}$ of bandwidth centered around $800 \mathrm{~nm}$, to a chirped pulse regenerative amplifier (Coherent Legend Elite USP) at an $80 \mathrm{MHz}$ repetition rate. The amplifier produces $4 \mathrm{~mJ}, 37$ fs transform-limited pulses centered around $800 \mathrm{~nm}$ at a $1 \mathrm{kHz}$ repetition rate. A 99\% reflective dielectric mirror acts as a 99:1 beam splitter to divide the amplifier's output into a $\mathrm{THz}$ generation beam, and a $\mathrm{THz}$ detection beam.

The 99\% split of the laser's output provides the spectrometer's THz light via a two-color plasma. To make this plasma, a betabarium borate (BBO) crystal (Eksma Optics), cut for Type 1 second harmonic generation (SHG) at $800 \mathrm{~nm}$, frequency doubles some of the $800 \mathrm{~nm}$ light to $400 \mathrm{~nm}$. A birefringent calcite plate (Eksma Optics) then corrects for the normal group velocity dispersion between 800 and $400 \mathrm{~nm}$. Both colors pass through a zero-order dual-wavelength wave plate (Eksma Optics \#465-4211) to align the polarizations of the 800 and $400 \mathrm{~nm}$ light. Finally, a gold-coated OAPM focuses both colors of light co-linearly in an environment purged with $\mathrm{N}_{2}(\mathrm{~g})$. The resulting oscillating plasma produces a broad-bandwidth, intense $\mathrm{THz}$ pulse, with the same polarization as the optical light fields. ${ }^{51-54}$ Another OAPM focuses the $\mathrm{THz}$ pulse down onto the ice sample in the vacuum chamber. An optical chopper placed before the BBO crystal modulates the $\mathrm{THz}$ generation beam at $100 \mathrm{~Hz}$ for lock-in detection.

We employ free-space, electro-optic sampling to detect the THz light transmitted through the ice sample. ${ }^{55,56}$ Optics route the remaining $1 \%$ of the $800 \mathrm{~nm}$ light from the original $4 \mathrm{~mJ}$ pulse down an opto-mechanical delay line (Newport ISL 150 with ESP300 controller) to change the relative delay between the $\mathrm{THz}$ generation and detection beams. The detection beam then passes through a half-wave plate (Newport 10RP02-46) which can be rotated to ensure that the linear polarization of the detection beam, and of the $\mathrm{THz}$ beam are matched at the detector crystal for maximum signal. An indium tin oxide (ITO) coated glass then serves to recombine the detection beam with the $\mathrm{THz}$ beam by reflecting the THz light and transmitting the $800 \mathrm{~nm}$ detection light so that the two beams co-axially propagate. An OAPM focuses the recombined beams onto a $\mathrm{GaP}(110)$ detector crystal (Incrys, $10 \mathrm{~mm} \times 10 \mathrm{~mm} \times 0.4 \mathrm{~mm})$. The electric field of the $\mathrm{THz}$ light interacts with the crystal to produce a DC-bias, as the THz field is effectively DC when compared to optical frequencies. When $800 \mathrm{~nm}$ light co-propagates with $\mathrm{THz}$ light in the crystal, the $\mathrm{THz}$ electric field rotates the polarization of the $800 \mathrm{~nm}$ light via the Pockels effect. The magnitude of this change in polarization is proportional to the magnitude of the THz electric field applied. After passing through the detector crystal, a lens refocuses the $800 \mathrm{~nm}$ beam to ensure that the photodiodes detect all the light. It then propagates through a quarter-wave plate (Newport 10RP04-46) to create a circularly polarized beam that is split into orthogonal polarizations by a Wollaston Prism (Thorlabs WP20). The signal is measured as a difference signal generated by a pair of balanced photodiodes (Thorlabs PDA36A). When no THz light is incident on the GaP crystal, the photodiodes read the same voltage, but when $\mathrm{THz}$ light is present, the photodiodes are no longer balanced, and the resulting difference signal is proportional to the THz electric field.

To measure the entire THz waveform in the time domain, the delay line moves to increase the detection path length so that the detection pulse interacts with the THz field at a different point in time. A digital lock-in amplifier (Stanford Research Systems SR830) records the spectrum as a function of delay. An example time domain trace collected with no sample on the substrate can be seen in Fig. 3. The spectrum is an average of 32 co-added scans, with a $100 \mathrm{~ms}$ time constant on the lock-in amplifier. The peak-to-peak signal-to-noise ratio (SNR) of a single scan collected with the lock-in amplifier in the time domain is $325: 1$.

Using a plasma source and electro-optic (EO) sampling with a GaP detector crystal, the Caltech TD THz spectrometer detects a pulsed THz electric field with frequency content from $0.3 \mathrm{THz}$ to $7.5 \mathrm{THz}$. The bandwidth can be seen in Fig. 4, which is a numerical fast-Fourier transform (FFT) of the data in Fig. 3, and the figure also shows the overlap of our spectrometer with the

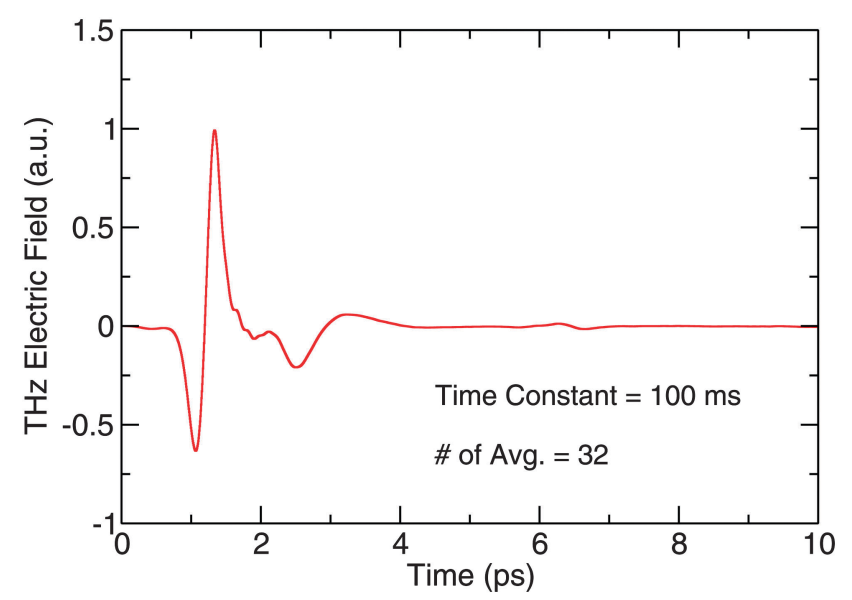

Fig. $3 \mathrm{~A} \mathrm{THz}$ trace of the blank substrate collected by the TD THz spectrometer. 


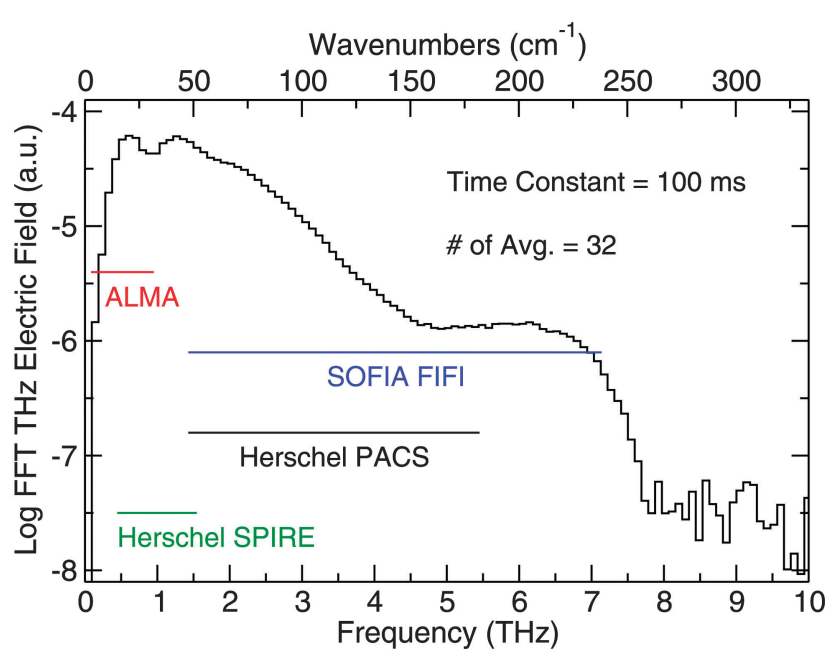

Fig. 4 The bandwidth of the THz spectrometer. The ordinate provides information on the relative strength of the $\mathrm{THz}$ electric field within the $\mathrm{THz}$ pulse at different frequencies. The horizontal lines indicate the spectral coverage of observational facilities in the $\mathrm{THz}$ region.

capabilities of some of the aforementioned sub-millimeter and far-IR telescopes. In addition, Fig. 4 shows the SNR in the frequency domain. By comparing the magnitude of signal on the ordinate to the noise level, which corresponds to the structure beyond 7.5 THz, one can roughly see how the SNR breaks down over the bandwidth of the spectrometer. In the 0.5 to $1.5 \mathrm{THz}$ region, we report a SNR of $1800: 1$. From 1.5 to $4.5 \mathrm{THz}$, the SNR decreases by a factor of roughly 3 per $100 \mathrm{GHz}$ starting at $1800: 1$ until it reaches $30: 1$, and the SNR remains flat at that value from 4.5 to $6.5 \mathrm{THz}$. The SNR then falls off again from 6.5 to $7.5 \mathrm{THz}$, where the signal meets the noise level.

The length of the scan in the time domain limits the resolution of the data in the frequency domain. While in theory only the length of the delay line limits the frequency-domain resolution, etalons produced in the EO detector crystal, and other optics through which the $\mathrm{THz}$ light passes, can add structure to the data. Small amounts of drift in the frequency position of the etalons over the course of an experimental run can be caused by environmental fluctuations and can change the etalon positions in the sample relative to the background scan. The etalons will then not divide out during background subtraction because of the time required to collect a spectrum. While there exist ways to push etalon peaks further away from the main THz pulse or remove them entirely, alternatively, one can scan a smaller window in the time domain to avoid these recurrences of the THz pulse. In this work, we co-added 32 spectra in the time domain to create an average which we subsequently used to calculate a FFT using boxcar apodization and zero-padding the length to the next power of 2 . The spectra were collected over a $10 \mathrm{ps}$ window in the time domain, chosen to avoid etalon features, which corresponds to a frequency domain resolution of $94 \mathrm{GHz}$ $\left(3 \mathrm{~cm}^{-1}\right)$. The sample spectra were then divided by a reference scan of the bare Si substrate to provide the absorption spectra shown in the following figures.

The spectral coverage of the combined $\mathrm{THz}$ and FTIR capabilities can be seen in Fig. 5 . The spectrometer coverage

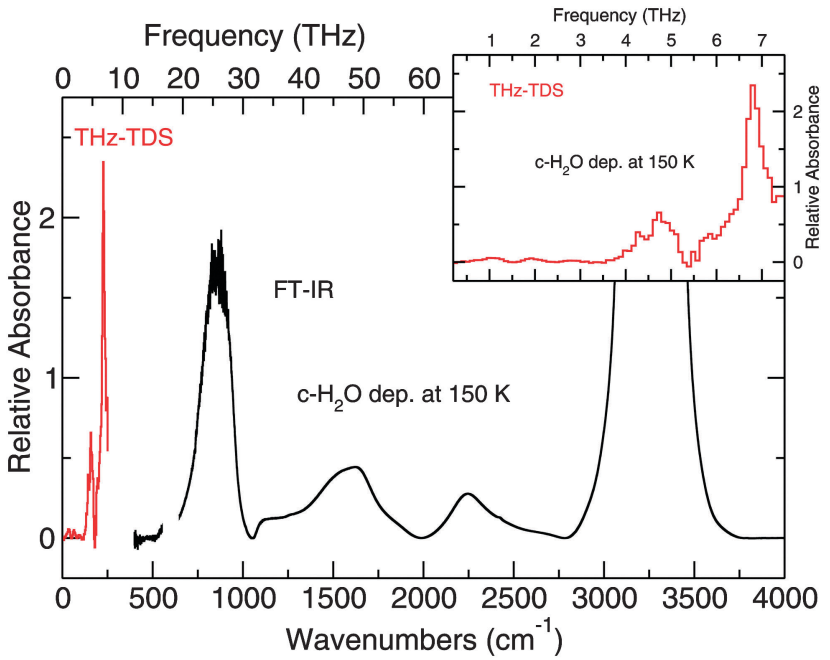

Fig. 5 The spectrum of crystalline $\mathrm{H}_{2} \mathrm{O}$ ice deposited at $150 \mathrm{~K}$ and collected at $150 \mathrm{~K}$. This spectrum demonstrates the complete spectral window covered by the spectrometer. The inset shows a zoom of the region collected with the THz-TDS.

extends from $10-4000 \mathrm{~cm}^{-1}$ with a gap from $250-400 \mathrm{~cm}^{-1}$ and a small gap between $580-630 \mathrm{~cm}^{-1}$ because the signal falls below the noise in those ranges. Using this broad spectral coverage, we are able to draw conclusions about the ices from both the $\mathrm{THz}$ and mid-IR regions.

\section{Results and discussion}

We present the results in three subsections. THz and mid-IR results for pure ices of $\mathrm{H}_{2} \mathrm{O}$ and $\mathrm{CO}_{2}$ are discussed first. Identifying the features and structure of pure ices allows us to understand how such features change when molecules are mixed or layered. Second, results from experiments of $\mathrm{H}_{2} \mathrm{O}: \mathrm{CO}_{2}$ mixed ices that are co-deposited (CD) are discussed. Finally, data collected from layered ice samples are presented. The layered experiments performed are crystalline sequential (CS) deposition, amorphous sequential (AS) deposition, and inverse sequential (IS) deposition, where we have used the nomenclature established in the literature. ${ }^{36}$ Table 1 summarizes the experiments discussed below.

All ices deposited in this study fall into the "thick" regime (>100 monolayers) and are approximately $0.5-5 \mu \mathrm{m}$ thick for $\mathrm{H}_{2} \mathrm{O}$ ice. $\neq$ To help ensure consistent thickness, we used the height of the $\mathrm{OH}$ librational band between $700-900 \mathrm{~cm}^{-1}$ to asses when to stop deposition of water. The following figures show that the relative thickness of the different ices is comparable. These experiments and others in the cryostat ${ }^{60}$ have shown that the ice is stable for at least $24 \mathrm{~h}$ as long as the temperature is kept

$\ddagger$ To estimate the ice thickness, we first obtained the column densities for $\mathrm{H}_{2} \mathrm{O}$ and $\mathrm{CO}_{2}$ from the integrated intensity of their IR bands using a modified Lambert-Beer equation. We selected the $\mathrm{O}-\mathrm{H}$ bending mode for $\mathrm{H}_{2} \mathrm{O}\left(1660 \mathrm{~cm}^{-1}\right)$ and the $\mathrm{O}-\mathrm{C}-\mathrm{O}$ bending mode for $\mathrm{CO}_{2}\left(660 \mathrm{~cm}^{-1}\right)$, and used the relevant absorption band strength as reported in Gerakines et al. $1995 .{ }^{57}$ The ice thickness is subsequently estimated by dividing the column density of each species by a conversion factor that is proportional to the density of the species in the ice. The density of $\mathrm{H}_{2} \mathrm{O}$ used is $0.9 \mathrm{~g} \mathrm{~cm}^{-3},{ }^{58}$ and the density of $\mathrm{CO}_{2}$ used is $1.5 \mathrm{~g} \mathrm{~cm}^{-3}$. ${ }^{59}$ 
Table 1 List of experiments performed in this work

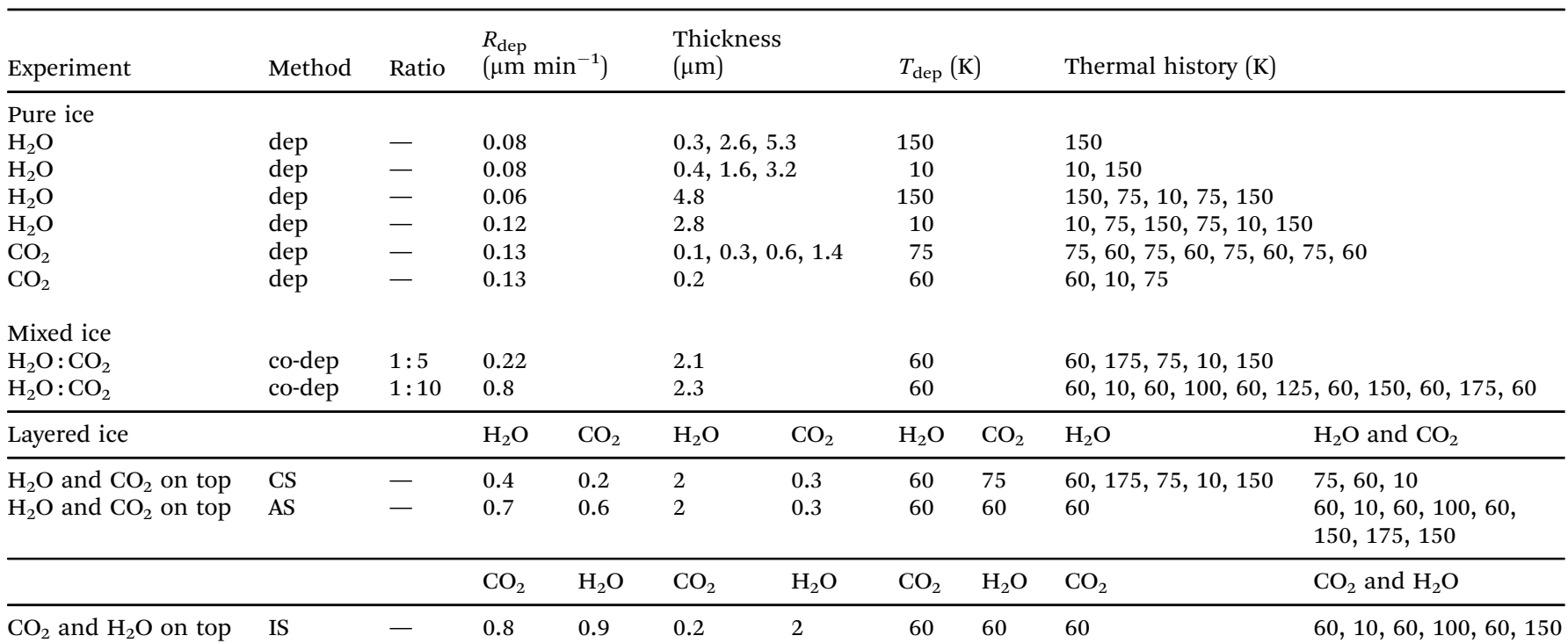

Deposition of single species (dep), co-deposition (co-dep), and sequential deposition (CS, AS, and IS) are performed under different laboratory conditions; $R_{\text {dep }}$ is the deposition rate of a selected molecule expressed in $\mu \mathrm{m} \min ^{-1}$; thickness is expressed in $\mu \mathrm{m}$; $T_{\mathrm{dep}}$ is the substrate temperature during deposition; thermal history is the temperatures that the ice have been sequentially exposed to. The heating ramp rate is $20 \mathrm{~K} \mathrm{~min}^{-1}$, in all cases.

below the sublimation temperature of a given ice. Moreover, the residual gasses in the vacuum chamber consist mainly of $\mathrm{H}_{2} \mathrm{O}$, accreting at a rate of roughly $1 \times 10^{12}$ molecules $\mathrm{cm}^{-2} \mathrm{~s}^{-1}$ $\left(0.001 \mu \mathrm{m} \mathrm{h}^{-1}\right)$ when the substrate is kept at $10 \mathrm{~K}$. Thus, relative to the ice thickness used in our study, the background water deposition can be considered negligible over the course of the experiment.

It must be noted here that in all of the following $\mathrm{THz}$ spectra, there is noise at $3.2 \mathrm{THz}$ that is only the width of one frequency bin. This noise spike appears to be some form of laser noise that we were unable to filter out while conducting these experiments. As such, the point corresponding to this noise feature is not plotted in most of the following spectra.

\subsection{Spectral fitting of the $\mathrm{THz}$ data}

To quantify the changes in the structure of mixed ices relative to the structure of pure ices samples, a spectral fitting procedure was used. The procedure makes use of a singular value decomposition to compare how the $\mathrm{THz}$ spectra of the mixtures differ from those of the pure ices. A set of four pure $\mathrm{H}_{2} \mathrm{O}$ ice spectra forms the basis of components used to create a fit (Fig. 6). The spectrum of ASW was collected at $10 \mathrm{~K}$ from an ice that was deposited at $10 \mathrm{~K}$. For the spectra of crystalline $\mathrm{H}_{2} \mathrm{O}$, a pure $\mathrm{H}_{2} \mathrm{O}$ ice was deposited at $150 \mathrm{~K}$ and then cooled to $75 \mathrm{~K}$ and $10 \mathrm{~K}$, with spectra collected at each of these temperatures. The coefficients resulting from the singular value decomposition reflect the weight of a given spectrum in the fit. The fits can be seen plotted in the figures that follow of mixed and layered ices.

To normalize the fit coefficients for experiment-to-experiment differences in the ices, the coefficients for a given component are multiplied by the integrated area of the mid-IR $\mathrm{H}_{2} \mathrm{O}$ bending mode at $\sim 1600 \mathrm{~cm}^{-1}$ in the pure ice spectrum for that component. The integrated area of the bending mode of the ice mixture

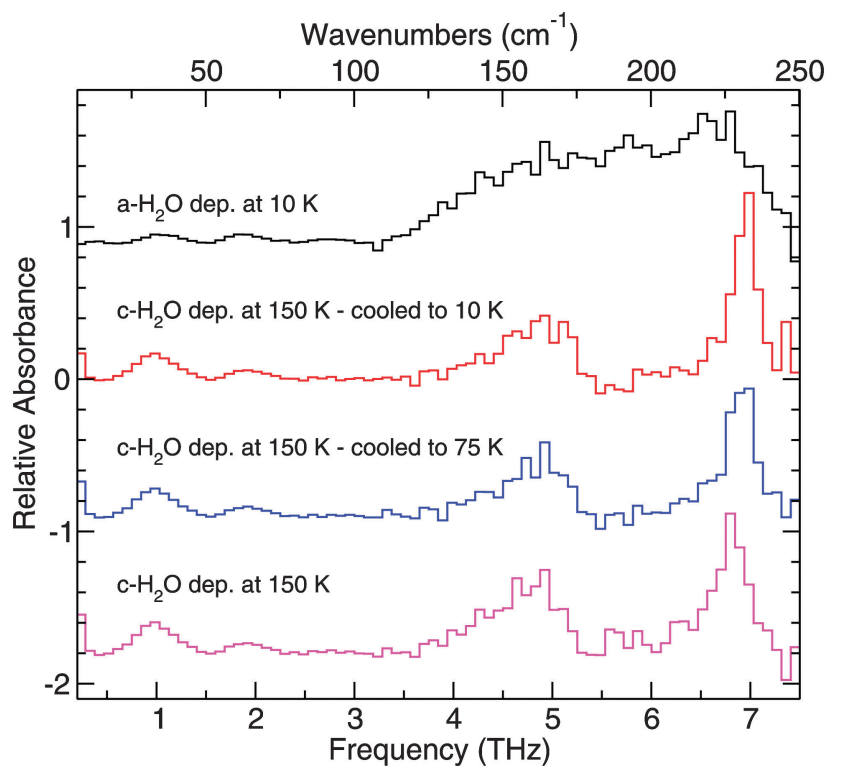

Fig. 6 Component spectra used to create the fits to the $\mathrm{THz}$ data. The top spectrum corresponds to the $\mathrm{THz}$ spectra of amorphous $\mathrm{H}_{2} \mathrm{O}$ ice, while the bottom three spectra correspond to crystalline $\mathrm{H}_{2} \mathrm{O}$ ice. The spectra are labeled with their deposition temperature and temperature at which the spectra were collected. For the top and bottom traces, the spectra were collected at the deposition temperature.

being fit defines the value for $100 \%$ area. The coefficients of the fit components are reported as a percentage of the total area of the $\mathrm{H}_{2} \mathrm{O}$ bending mode of a given ice mixture being analyzed.

The degree to which the sum of the normalized coefficient percentages approaches $100 \%$ indicates the ability of the four component basis to reproduce the spectra of the mixtures being analyzed. In cases where the structure of the mixed or layered 
ice is not expected to differ from the pure ices structure, such as in the CS deposition, the sum of the fit coefficient percentages are close to $100 \%$. In some cases, however, the sum of the fit coefficient percentages does not approach $100 \%$. This does not signify that the fit procedure works poorly, since the fit still qualitatively reproduces the spectral data - instead, it shows that the structure of that ice differs somehow from the structure of the pure ice components. When the fit components of a given ice sum to a percentage much less than $100 \%$, that ice cannot be described by a basis consisting only of pure $\mathrm{H}_{2} \mathrm{O}$ ice components. Thus, a poor fit actually provides useful information about what the structure of the ice does not look like. In a few specific cases, such as in the CD $60 \mathrm{~K} / 125 \mathrm{~K}$ and the CD $60 \mathrm{~K} / 175 \mathrm{~K}$, the procedure was incapable of qualitatively fitting the data accurately and thus shows that the structure of the ice being fit differs significantly from the structure of a pure ice. However, since the fit was unsuccessful, there is no value in trying to quantify the difference from the structure of a pure ice, and so fit percentages are not reported for these data. The specific fits are discussed in more detail in the context of the specific experiments below.

\subsection{Pure ices}

Pure $\mathrm{H}_{2} \mathrm{O}$ has been deposited in two different forms, amorphous solid water (ASW) and crystalline $\mathrm{H}_{2} \mathrm{O} .{ }^{61}$ Cooling the substrate down below $100 \mathrm{~K}$ before deposition ensures deposition of porous ASW, which lacks a well-defined long-range crystal structure. Crystalline $\mathrm{H}_{2} \mathrm{O}$ can be formed in two ways. If the substrate is kept above $140 \mathrm{~K}$, crystalline $\mathrm{H}_{2} \mathrm{O}$ ice will form upon deposition. Another way to form crystalline $\mathrm{H}_{2} \mathrm{O}$ is to deposit ASW at temperatures less than $100 \mathrm{~K}$, which is then annealed. Above $140 \mathrm{~K}$, ASW undergoes a non-equilibrium transition to the more thermodynamically stable, crystalline $\mathrm{H}_{2} \mathrm{O}$ form.

Fig. 6 highlights the THz spectroscopic differences between ice structures formed at different deposition temperatures. Both crystalline and amorphous $\mathrm{H}_{2} \mathrm{O}$ have an absorption feature at $1.9 \mathrm{THz}$. ASW resulting from deposition at $10 \mathrm{~K}$ has one broad absorption feature starting at approximately $3.1 \mathrm{THz}$ and extending out past 7.5 THz. In the corresponding region for crystalline $\mathrm{H}_{2} \mathrm{O}$, deposited at $150 \mathrm{~K}$, distinct peaks with the following assignments ${ }^{40,62}$ are seen: 6.8 THz (hydrogen-bonded bilayer stretch), 5.7 THz (out-ofphase vibration within the bilayer), $4.7 \mathrm{THz}$ (proton disordered motion), 4.2 THz, and 1.9 THz (corresponding to an $\mathrm{O}-\mathrm{O}-\mathrm{O}$ bend) which are in good agreement with theory and previous experiments. ${ }^{43,63-65}$ The peak positions of the THz features for the pure ices studied are summarized in Table 2.

In addition to the features described above, a feature appears in these spectra at $1 \mathrm{THz}$ that has not been previously observed in far-IR experiments, and so we tentatively report a new feature at $1 \mathrm{THz}$. This feature has likely not been seen in previous work because a TD THz spectrometer, like the one used in this study, has a higher signal-to-noise around this band than a comparable far-IR FTIR study. Over the 0.1 to $3 \mathrm{THz}$ region, a plasma emitter produces more photons than the incoherent source in an FTIR, and so features which would have been below the noise level of the previous work can be detected with our TD THz spectrometer. As such, previous experiments did not extend below $50 \mathrm{~cm}^{-1}$ and were thus unable to see this feature.
Table 2 The peak positions of pure $\mathrm{H}_{2} \mathrm{O}$ ice absorption features in the $\mathrm{THz}$ region in frequency $(\mathrm{THz})$ and wavenumber

\begin{tabular}{|c|c|c|c|}
\hline \multicolumn{4}{|l|}{$\underline{\mathrm{H}_{2} \mathrm{O}}$} \\
\hline \multicolumn{2}{|c|}{ Amorphous } & \multicolumn{2}{|c|}{ Crystalline } \\
\hline THz & $\mathrm{cm}^{-1}$ & $\mathrm{THz}$ & $\mathrm{cm}^{-1}$ \\
\hline 1.0 & 33 & 1.0 & 33 \\
\hline 1.9 & 63 & $1.9^{a}$ & 63 \\
\hline \multirow[t]{4}{*}{5.5} & 181 & $4.2^{e}$ & 139 \\
\hline & & $4.7^{b}$ & 155 \\
\hline & & $5.7^{c}$ & 188 \\
\hline & & $6.8^{d}$ & 224 \\
\hline
\end{tabular}

The transitions of $\mathrm{H}_{2} \mathrm{O}$ ice can be assigned as follows: ${ }^{a} \mathrm{O}-\mathrm{O}-\mathrm{O}$ bend. ${ }^{b}$ Proton disordered vibration. ${ }^{c}$ Out-of-phase vibration of molecules within a bilayer. ${ }^{d}$ Hydrogen bond stretch between bilayers. ${ }^{e}$ The feature at $4.2 \mathrm{THz}$ is blended with the feature at $4.7 \mathrm{THz}$.

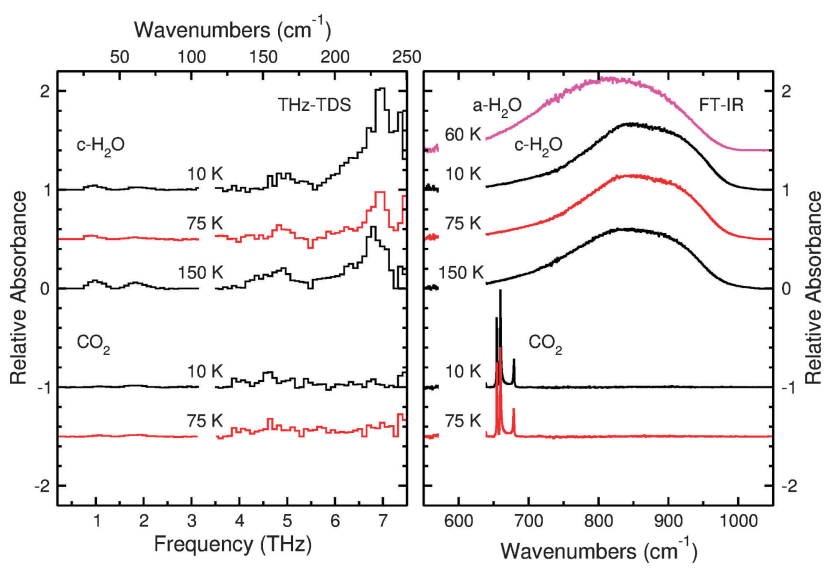

Fig. $7 \mathrm{THz}$ spectra (left panel) and mid-IR spectra (right panel) of pure crystalline $\mathrm{H}_{2} \mathrm{O}$ and pure $\mathrm{CO}_{2}$ at different temperatures. Both molecules were deposited at $60 \mathrm{~K}$ and then the ices were annealed. The $\mathrm{H}_{2} \mathrm{O}$ was annealed at $175 \mathrm{~K}$, while the $\mathrm{CO}_{2}$ was annealed at $75 \mathrm{~K}$.

The results of the other method of preparing crystalline $\mathrm{H}_{2} \mathrm{O}$ are shown in Fig. 7. In contrast with Fig. 6, where crystalline water ice was prepared by deposition at $150 \mathrm{~K}$, the crystalline $\mathrm{H}_{2} \mathrm{O}$ ice presented in Fig. 7 was deposited as ASW at $60 \mathrm{~K}$, then annealed to $175 \mathrm{~K}$ and subsequently cooled. In these experiments, $\mathrm{H}_{2} \mathrm{O}$ transitions from compact amorphous to crystalline ice start at $\sim 140 \mathrm{~K}$, in good agreement with the literature. Annealing the ice to $175 \mathrm{~K}$ should ensure that the amorphous to crystalline transition is fully completed. ${ }^{66}$ There will be some mass lost from the ice when annealing at such high temperatures. However, because of the thickness of the ice, such loss is negligible. Under these vacuum conditions, $\mathrm{H}_{2} \mathrm{O}$ molecules start to desorb from the ice at $\sim 170 \mathrm{~K}$.

The $\mathrm{H}_{2} \mathrm{O}$ spectra in both Fig. 6 and 7 illustrate the spectral changes as a function of temperature. As the cryostat cools the ice sample, the $\mathrm{THz}$ feature that peaks at $6.8 \mathrm{THz}$ becomes sharper and more intense at $10 \mathrm{~K}$ relative to the same feature at $150 \mathrm{~K}$ because cooling the sample will reduce the thermal population of hot-band states of the vibration. The same is true for the shoulder at 5.7 THz. These changes in shape may be useful for determining the temperature of an ice detected in space from its $\mathrm{THz}$ spectrum. 
Fig. 7 shows the absorption spectrum for pure $\mathrm{CO}_{2}$ ice. Although Moore and Hudson reported that $\mathrm{CO}_{2}$ should have one narrow feature at $3.3 \mathrm{THz},{ }^{43}$ we observe no unambiguous features in our spectra. This is likely caused by a combination of sensitivity and, especially, resolution, owing to the proximity of this feature to the noise spike at $3.2 \mathrm{THz}$ in our experiments. Molecules of $\mathrm{CO}_{2}$ begin to desorb at $\sim 90 \mathrm{~K}$ from the ice, and an ice of pure $\mathrm{CO}_{2}$ will be fully desorbed at $100 \mathrm{~K}$ under these experimental conditions. In the following sections, we will show that the desorption temperature of $\mathrm{CO}_{2}$ varies with respect to the ice composition.

In conjunction with the THz spectra, the right panel of Fig. 7 also shows the mid-IR spectra collected with the FTIR spectrometer from the same samples. This portion of the spectrum shows the librational mode of water ice and is a useful region of the spectrum to consider because resonances corresponding to the bending mode of $\mathrm{CO}_{2}$ also appear in this same spectral window. A spectrum of ASW is presented first for comparison, and one can easily see the differences in shape between the amorphous and crystalline ice. The ASW peak is more symmetric than that of crystalline $\mathrm{H}_{2} \mathrm{O}$, and this asymmetry becomes more pronounced as the crystalline ice cools from 150 to $10 \mathrm{~K}$. These FTIR spectra are also useful because $\mathrm{CO}_{2}$ shows clear resonances distinct from those of $\mathrm{H}_{2} \mathrm{O}$ ice. There are four peaks which appear in the spectrum of $\mathrm{CO}_{2}$. The two main peaks centered around $660 \mathrm{~cm}^{-1}$ are the main features of the $\mathrm{CO}_{2}$ bending mode for S-polarized light. The peak at $680 \mathrm{~cm}^{-1}$ is the signature of the $\mathrm{CO}_{2}$ bending mode for P-polarized light. Given that the angle of incidence of the IR beam is $45^{\circ}$ and that there is no polarizer along the beam path, the FTIR spectra in this work will show contributions from both polarizations of light. The final peak centered around $640 \mathrm{~cm}^{-1}$ is much weaker, and it corresponds to the ${ }^{13} \mathrm{CO}_{2}$ bending mode. Analysis of FTIR data for $\mathrm{CO}_{2}$ allows for an effective determination of whether a mixed ice sample still contains small amounts of $\mathrm{CO}_{2}$ since the $\mathrm{THz}$ spectra of this molecule do not provide distinct and strong absorption features.

\subsection{Co-deposition of mixed ices}

The importance of $\mathrm{CO}_{2}$ in this study stems from how its presence affects the structure of $\mathrm{H}_{2} \mathrm{O}$ ice. In the ISM, such a mixture could arise from the formation of $\mathrm{CO}_{2}$ within a polar ice. ${ }^{67}$ Consequently, it is interesting to know if and how the presence of $\mathrm{CO}_{2}$ would affect the structure of the ice in which it exists.

Directly annealed mixture. Fig. 8 presents the data from two different experiments. In the top three spectra of the figure, a $\mathrm{H}_{2} \mathrm{O}: \mathrm{CO}_{2}$ mixture was prepared in the dosing line and deposited onto the substrate kept at $60 \mathrm{~K}$. Immediately following deposition, the ice was annealed to $175 \mathrm{~K}$ at a rate of $20 \mathrm{~K}$ per minute and held at that temperature for several minutes. While held at that temperature, monitoring with the FTIR spectrometer showed some mass is lost from the deposited sample mostly from $\mathrm{CO}_{2}$ desorption. The sample was then cooled and spectra collected at $75 \mathrm{~K}$ and $10 \mathrm{~K}$, and then finally warmed to $150 \mathrm{~K}$ where spectra were acquired. The peaks of the $\mathrm{H}_{2} \mathrm{O}$ bending mode and the $\mathrm{CO}_{2}$ bending mode at 1600 and $600 \mathrm{~cm}^{-1}$, respectively, are integrated and, using the relevant absorption

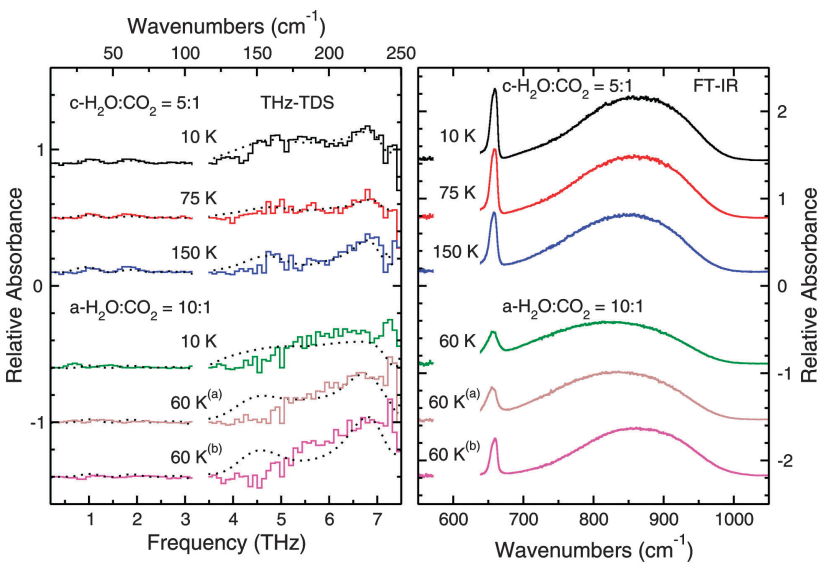

Fig. $8 \mathrm{THz}$ spectra (left panel) and mid-IR spectra (right panel) of mixed $\mathrm{H}_{2} \mathrm{O}$ and $\mathrm{CO}_{2}$ ice co-deposited. Data for mixed ices that were annealed directly after deposition are presented in the top three traces of the figure. The spectra were collected after cooling. In the bottom three traces, the spectra collected from a mixed ice that was cooled immediately after deposition and stepwise annealed are presented. The spectra are labeled with the temperature at which they were collected. The dashed lines in the plot correspond to the fits calculated for the data from the spectra of the pure species, which are plotted with a polynomial spline fit for clarity. Note that in the bottom traces, the effect of the $\mathrm{CO}_{2}$ on the $\mathrm{H}_{2} \mathrm{O}$ ice structure can be seen from the blue shift of the ASW feature, and after annealing, the 4.5 THz transition is missing from the spectrum. The inability of the fits to reproduce the spectra show that the structure of the ices differ greatly from the structure of pure ices. The components of the fits are summarized in Table 3. The superscripts denote the temperature to which the ices were annealed: ${ }^{a}$ annealed to $125 \mathrm{~K},{ }^{b}$ annealed to $175 \mathrm{~K}$.

band strengths, ${ }^{57}$ we determine that we have a mixture with a $\mathrm{H}_{2} \mathrm{O}: \mathrm{CO}_{2}$ ratio of $\sim 5: 1$.

Annealing at such a high temperature should force the water to become completely crystalline, ${ }^{66}$ and one can see that the spectra of the mixture exhibit $\mathrm{THz}$ features that are quite similar to crystalline $\mathrm{H}_{2} \mathrm{O}$ at $10 \mathrm{~K}$ and $75 \mathrm{~K}$ but differing in intensity and structure. Compared with the spectrum of pure crystalline $\mathrm{H}_{2} \mathrm{O}$ ice at $75 \mathrm{~K}$, the $6.8 \mathrm{THz}, 5.7 \mathrm{THz}$, and $4.7 \mathrm{THz}$ features are not as intense in the spectrum of this ice. Even at $10 \mathrm{~K}$, where the pure ice features are sharpest and most intense, the $6.8 \mathrm{THz}$ feature in Fig. 8 does not appear to change compared to the spectrum at $75 \mathrm{~K}$.

The changes in these transitions results from the presence of $\mathrm{CO}_{2}$ in the ice. One can see in the right panel of Fig. 8 the resonance corresponding to the $\mathrm{CO}_{2}$ bending vibrational mode. The shape of this peak is qualitatively different from that of pure $\mathrm{CO}_{2}$ since now only one peak with a Lorentzian lineshape is observed as opposed to the three peaks observed previously. Fig. 8 shows that, even though the ice was annealed to a temperature above the $\mathrm{CO}_{2}$ sublimation temperature, not all of the $\mathrm{CO}_{2}$ molecules leave the ice. This effect has been previously observed by temperatureprogrammed desorption (TPD) experiments using a quadrupole mass spectrometer (QMS). ${ }^{68}$ As a result of the co-deposition of the two molecules, some $\mathrm{CO}_{2}$ molecules remain trapped by the molecules deposited on top of them. We thus observe the "interior" $\mathrm{CO}_{2}$ as discussed previously in the literature. ${ }^{36-38}$ Clearly, after annealing, the trapped $\mathrm{CO}_{2}$ molecules affect the 
ability of the $\mathrm{H}_{2} \mathrm{O}$ molecules to reorient themselves to form long-range order within the solid ice and properly crystallize, but the observed structure does differ from that of pure ASW.

By comparing the mid-IR data given in the top three spectra in Fig. 8 with the top three spectra in Fig. 7 (right panel), it is possible to conclude that the $\mathrm{H}_{2} \mathrm{O}$ lacks a fully crystalline structure. However, from the $\mathrm{THz}$ spectra alone, the lack of crystalline $\mathrm{H}_{2} \mathrm{O}$ features is also clear. In addition, given that features in the $\mathrm{THz}$ region involve the longer range coordinated motion of many molecules, the $\mathrm{THz}$ data also provide insight into the location of the $\mathrm{CO}_{2}$ molecules within the ice by observing which spectral features are disrupted.

To quantitatively characterize the structure of the ice, we fitted the THz spectral profile of the features shown in Fig. 8 as discussed in Section 3.1. The fits to these spectra plotted in Fig. 8 agrees well qualitatively with the spectra of the mixed ice. The fits show that the pure $\mathrm{H}_{2} \mathrm{O}$ bands at 4.7 and $5.7 \mathrm{THz}$ are blueshifted in the spectra of the mixtures while the $6.8 \mathrm{THz}$ feature is always fit well by using a spectrum of pure $\mathrm{H}_{2} \mathrm{O}$ ice acquired at the same temperature as the mixed ice. In all of these cases, a fit component corresponding to ASW was needed to improve the fit. The specific fit components for each spectrum are summarized in Table 3 . Since the percentages listed in the table do not approach $100 \%$, they provide another piece of evidence showing that the structure of these ices differs from the structure of pure crystalline water ice.

Cooled before annealed mixture. The bottom set of spectra in Fig. 8 correspond to a mixed ice prepared similarly to the ice

Table 3 The components of pure crystalline $\mathrm{H}_{2} \mathrm{O}$ and ASW ice features that comprise the fits to the experimental data

\begin{tabular}{|c|c|c|c|c|c|}
\hline \multirow[b]{2}{*}{ Experiments } & \multicolumn{5}{|c|}{ Fit component (percentage) } \\
\hline & $\begin{array}{l}\text { ASW } \\
10 \mathrm{~K}\end{array}$ & $\begin{array}{l}\mathrm{c}-\mathrm{H}_{2} \mathrm{O} \\
10 \mathrm{~K}\end{array}$ & $\begin{array}{l}\mathrm{c}-\mathrm{H}_{2} \mathrm{O} \\
75 \mathrm{~K}\end{array}$ & $\begin{array}{l}\mathrm{c}-\mathrm{H}_{2} \mathrm{O} \\
150 \mathrm{~K}\end{array}$ & $\operatorname{Total}^{a}(\%)$ \\
\hline $\mathrm{CD}^{b} 10 \mathrm{~K}$ & 59 & 18 & & & 77 \\
\hline $\mathrm{CD}^{b} 75 \mathrm{~K}$ & 21 & & 17 & & 38 \\
\hline $\mathrm{CD}^{b} 150 \mathrm{~K}$ & 20 & & & 47 & 67 \\
\hline $\mathrm{CD}^{c} 10 \mathrm{~K}$ & 69 & & & & 69 \\
\hline $\mathrm{CD}^{c} 60 \mathrm{~K} / 125 \mathrm{~K}^{d}$ & & & & & $\underline{e}^{e}$ \\
\hline $\mathrm{CD}^{c} 60 \mathrm{~K} / 175 \mathrm{~K}$ & & & & & $-^{e}$ \\
\hline CS $10 \mathrm{~K}$ & & 99 & & & 99 \\
\hline CS $60 \mathrm{~K}$ & & 99 & & & 99 \\
\hline AS $10 \mathrm{~K}$ & 95 & & & & 95 \\
\hline AS $60 \mathrm{~K}$ & 87 & & & & 87 \\
\hline AS $60 \mathrm{~K} / 100 \mathrm{~K}$ & 73 & & & 12 & 85 \\
\hline AS $150 \mathrm{~K}$ & 66 & & & 21 & 87 \\
\hline AS $150 \mathrm{~K} / 175 \mathrm{~K}$ & 61 & & & 22 & 83 \\
\hline IS $10 \mathrm{~K}$ & 33 & & & & 33 \\
\hline IS $60 \mathrm{~K}$ & 60 & & & & 60 \\
\hline IS $60 \mathrm{~K} / 100 \mathrm{~K}$ & 52 & & & 8 & 60 \\
\hline
\end{tabular}

${ }^{a}$ The percentage does not always equal $100 \%$ because the structure of the ice cannot always be described by the components of pure $\mathrm{H}_{2} \mathrm{O}$ used. ${ }^{b}$ These are the data for the CD experiments which were directly annealed. ${ }^{c}$ These are the data for the $\mathrm{CD}$ experiments which were cooled first and then stepwise annealed. ${ }^{d}$ In this table $60 \mathrm{~K} / 100 \mathrm{~K}$ is defined as a spectrum collected at $60 \mathrm{~K}$ for an ice that was first annealed to $100 \mathrm{~K}$. This convention holds for all lines in the experiments column where two temperature values are listed. ${ }^{e}$ No percentages are reported in this case since the fitting procedure produced a poor fit to the actual spectra. As such, a quantitative analysis is not meaningful. in the top three spectra. A $\mathrm{H}_{2} \mathrm{O}: \mathrm{CO}_{2}$ gaseous mixture, was prepared in the dosing line and deposited onto the substrate held at $60 \mathrm{~K}$. Similarly to the previous section, after deposition, the mid-IR $\mathrm{H}_{2} \mathrm{O}$ and $\mathrm{CO}_{2}$ bending modes were used to derive the column densities and determine that after deposition the ice mixture has a $\mathrm{H}_{2} \mathrm{O}: \mathrm{CO}_{2}$ ratio of $\sim 10: 1$. In this set of experiments, the ice was first cooled to $10 \mathrm{~K}$ and a spectrum was collected. Then the ice was warmed back up to the deposition temperature of $60 \mathrm{~K}$. The spectra at $10 \mathrm{~K}$ and $60 \mathrm{~K}$ agree qualitatively with the shape of the spectrum of pure, ASW in Fig. 6, however, the relative absorbance of this mixed ice does not appear as strong as that of the pure $\mathrm{H}_{2} \mathrm{O}$. We can see from the mid-IR data in Fig. 8 that both $\mathrm{CO}_{2}$ and $\mathrm{H}_{2} \mathrm{O}$ are present in this ice. It is worth noting that the shape of the mid-IR $\mathrm{CO}_{2}$ bending mode in these unannealed spectra differs qualitatively from the shape of the bending mode in the annealed spectra at the top of the figure. The peak in the unannealed spectrum appears to have a flat top as opposed to a smooth curve and is redshifted relative to the annealed spectrum. Also, the $\mathrm{H}_{2} \mathrm{O}$ librational mode appears broader in the unannealed spectra than in the annealed spectra.

This ice was then annealed. First, the ice was annealed to $100 \mathrm{~K}$ and cooled down to $60 \mathrm{~K}$. Both the THz and FTIR spectra show no appreciable change compared to the unannealed spectra at $60 \mathrm{~K}$. The ice was then subsequently annealed to $125 \mathrm{~K}, 150 \mathrm{~K}$, and $175 \mathrm{~K}$, with a cool down cycle returning the ice to $60 \mathrm{~K}$ each time after annealing. There is no significant change to the ice after annealing until the ice is annealed up to $175 \mathrm{~K}$. However, the THz spectrum of the ice after annealing to $175 \mathrm{~K}$ does not match the spectrum taken of the ice that was directly annealed. As discussed above, the THz spectra of the ices that were directly annealed after deposition show features of crystalline $\mathrm{H}_{2} \mathrm{O}$. However, the spectra of the ice that was cooled before being annealed show features that correspond more to the structure of ASW.

A comparison between the bottom set of spectra in Fig. 8 and the spectral fits as discussed above shows that the presence of $\mathrm{CO}_{2}$ in the ice causes a blue shift of the broad band of amorphous water ice at $10 \mathrm{~K}$. Moreover, although the last spectrum annealed to $175 \mathrm{~K}$ shows a weak band around $6.8 \mathrm{THz}$ because of crystalline $\mathrm{H}_{2} \mathrm{O}$, the 4.7 and 5.7 THz bands are blue shifted and/or weak. In this case, and for all spectra annealed above $100 \mathrm{~K}$, the fit cannot reproduce the data satisfactorily. This shows that the structure of this ice is significantly different from the structure of the pure ices used in the fits.

Discussion of both ices. THz spectroscopy probes the intermolecular vibrations of an ice as opposed to the intramolecular vibrations which dominate the mid-IR. Different intermolecular interactions will, of course, affect the band profiles in a mid-IR spectrum. However, the features in the $\mathrm{THz}$ region that require the coordinated motion of many molecules will necessarily directly sense disruptions in the material structure and can disappear entirely from the $\mathrm{THz}$ spectrum if those interactions are removed.

The mid-IR FTIR data provide a strong basis for interpreting our THz spectra. None of the data in Fig. 8 (right panel) show the characteristic asymmetric feature of crystalline $\mathrm{H}_{2} \mathrm{O}$ in the $\mathrm{H}_{2} \mathrm{O}$ 
librational band (see Fig. 7), thus strengthening the interpretation that $\mathrm{CO}_{2}$ disrupts the crystallization process of the $\mathrm{H}_{2} \mathrm{O}$ ice. In addition, as previously mentioned, one can observe a difference in the features between the directly annealed spectra and the stepwise annealed spectra. With this information in hand, one can learn more about the structure of the ice from the $\mathrm{THz}$ spectra.

In particular, the THz region has the potential to indicate the degree of crystallization, and the history of transformations that an ice has undergone. Generally speaking, spectroscopy is not sensitive to the history of transformations an ice has undergone. However, if the different ices were to start at the same unstable equilibrium point and then undergo different, irreversible nonequilibrium transitions, the different histories would result in different spectra. For the ice that was annealed directly after deposition, we note that it does appear, from the THz spectra, to have some crystalline $\mathrm{H}_{2} \mathrm{O}$ structure. In this case, the $\mathrm{THz}$ spectra provide some insight into the degree of crystallinity. The ice was annealed to $175 \mathrm{~K}$, where one would have expected the crystallization process to be completed (see Fig. 7). The fact that its features are attenuated somewhat relative to the spectrum of pure crystalline $\mathrm{H}_{2} \mathrm{O}$ deposited at $60 \mathrm{~K}$ and annealed to $175 \mathrm{~K}$ likely results from the presence of segregated $\mathrm{CO}_{2}$ within the ice. Since it is known that segregation occurs quickly in thick ices, ${ }^{39}$ this ice most likely contains large regions of crystalline $\mathrm{H}_{2} \mathrm{O}$, but the pockets of segregated $\mathrm{CO}_{2}$ prevent a fully uniform crystalline solid from forming, and, thus, the peak strengths and shapes differ from that of pure crystalline $\mathrm{H}_{2} \mathrm{O}$.

In the other experiment, by cooling the ice immediately after deposition, the ice was taken below the temperature at which segregation can occur. While some segregation likely took place during the deposition process at $60 \mathrm{~K}$, by cooling first and stopping segregation, the $\mathrm{CO}_{2}$ is left more well mixed with $\mathrm{H}_{2} \mathrm{O}$. Then, by restarting the annealing process in steps, the $\mathrm{CO}_{2}$ molecules were forced to segregate more locally into smaller domains. In contrast, when the first ice was annealed directly after deposition at $60 \mathrm{~K}$, the segregation rate increased when the temperature was raised. This likely encouraged the formation of large domains of segregated $\mathrm{CO}_{2}$, and large regions composed entirely of $\mathrm{H}_{2} \mathrm{O}$ molecules. Consequently, the features of crystalline $\mathrm{H}_{2} \mathrm{O}$ are more pronounced in the directly annealed spectra when compared with the ice that was cooled immediately after deposition. This mechanism can potentially explain the differences observed in the $\mathrm{THz}$ spectra between the two sets of experiments.

The results from the quantitative fits discussed above provide further evidence to support the differences in the structure of the ices in the two different experiments. The spectra of the ice that was directly annealed can be fit quite well using these components as seen in Fig. 8. In contrast, the best fit of the stepwise annealed data still show a peak at $4.5 \mathrm{THz}$ meaning that using the fit components corresponding to pure $\mathrm{H}_{2} \mathrm{O}$ ice, the fit to the data is unsatisfactory. Consequently, the poor nature of the fit shows that the structure of the stepwise annealed ice differs significantly from the directly annealed ice which can be fit in this multicomponent fashion.

It would also be valuable to know the location of the segregated $\mathrm{CO}_{2}$ in the structure of the $\mathrm{H}_{2} \mathrm{O}$ ice. The $6.8 \mathrm{THz}$ feature in crystalline $\mathrm{H}_{2} \mathrm{O}$ corresponds to the stretching of hydrogen-bonded bilayers of $\mathrm{H}_{2} \mathrm{O}$ molecules. ${ }^{40}$ Since this feature is weak in the $\mathrm{THz}$ spectra of the ice that was cooled after deposition, the $\mathrm{CO}_{2}$ molecules must interfere with the hydrogen bonding between bilayers of crystalline $\mathrm{H}_{2} \mathrm{O}$ ice. Most likely, the $\mathrm{CO}_{2}$ molecules segregate together between bilayers and disrupt the $\mathrm{H}$-bond stretching that give rise to the feature at $6.8 \mathrm{THz}$. As discussed by Maté et al., ${ }^{37}$ "interior" $\mathrm{CO}_{2}$ must be located within some type of channels or pores within the ice. However, these authors were unable to determine specifically the location of "interior" $\mathrm{CO}_{2}$ within their $\mathrm{H}_{2} \mathrm{O}$ ice. By using $\mathrm{THz}$ spectroscopy and the known assignment of these attenuated features in the crystalline $\mathrm{H}_{2} \mathrm{O}$ ice, our results provide new insight into the specific location of the segregated $\mathrm{CO}_{2}$ within the $\mathrm{H}_{2} \mathrm{O}$ ice. The disruption of the feature at $6.8 \mathrm{THz}$ shows that the $\mathrm{CO}_{2}$ molecules segregate between the bilayers of the crystalline $\mathrm{H}_{2} \mathrm{O}$ ice structure.

We would be remiss if we did not discuss the previous work done in the far-IR on ice mixtures. Moore and Hudson in 1994 also co-deposited a mixture of $\mathrm{H}_{2} \mathrm{O}$ and $\mathrm{CO}_{2}$ and observed a disruption of the crystalline water features after annealing a co-deposited mixture. ${ }^{43}$ Our results, however, are an expansion on the previous work in two ways. First, we performed two different CD experiments, one which was directly annealed, and one which was stepwise annealed, and observed different results. Second, given recent theoretical advances in understanding crystalline $\mathrm{H}_{2} \mathrm{O}$ structure, ${ }^{40}$ and an improved knowledge of segregation dynamics, ${ }^{39}$ we can infer the position of the $\mathrm{CO}_{2}$ within the ice and provide new insight into the structure of ice mixtures by determining what types of pores and channels exist for the $\mathrm{CO}_{2}$ to occupy.

It must be noted that the ratio of $\mathrm{H}_{2} \mathrm{O}: \mathrm{CO}_{2}$ is different in the two ices discussed above, resulting from the experimental realities of deposition. In an attempt to achieve the same ratio in both ices, the mixing ratio in the dosing line was adjusted to try to account for the mass loss (predominantly of $\mathrm{CO}_{2}$ ) when the ice was annealed directly to $175 \mathrm{~K}$. However, the two ratios used were different by too large an amount, which led to the different mixtures observed in the ice. This results in a difference in the ice mixtures of less than a factor of two. Even considering these differences between the ices, the results of our study remain relevant. One might expect that a higher concentration of $\mathrm{CO}_{2}$ in $\mathrm{H}_{2} \mathrm{O}$ would disrupt the crystallization to a greater degree than having a lower concentration of $\mathrm{CO}_{2}$. However, our results indicate that crystallization of water occurs more readily in the directly annealed mixture that also has a slightly higher concentration of $\mathrm{CO}_{2}$ with respect to the stepwise annealed mixture. As such, we can conclude that segregation of $\mathrm{CO}_{2}$ still trapped in a $\mathrm{H}_{2} \mathrm{O}$ ice is influenced by the annealing procedure.

\subsection{Layered ices}

Layered ices present another interesting system where $\mathrm{THz}$ spectroscopy may provide some added insight into the longrange molecular structure. 


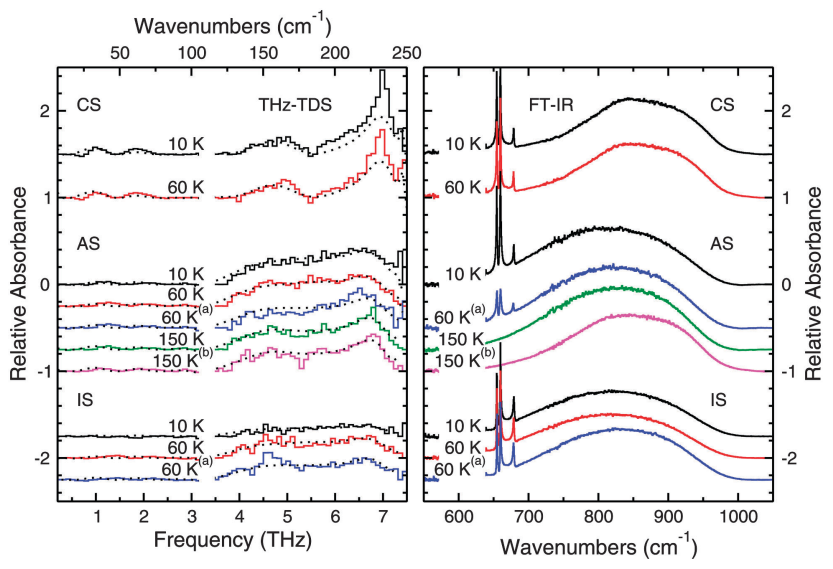

Fig. 9 THz spectra (left panel) and mid-IR spectra (right panel) of layered ice samples. The spectra collected from the crystalline sequential (CS) deposition are shown in the top two traces, those from the amorphous sequential (AS) deposition are shown in the middle traces. The spectra collected from the inverse sequential (IS) deposition are shown in the bottom three traces. All spectra are labeled according to the temperature at which they were collected and the conditions under which they were annealed. The dashed lines in the plot correspond to the fits calculated for the data from the spectra of the pure species, which are plotted with a polynomial spline fit for clarity. The superscripts denote thermal processing as follows: (a) annealed to $100 \mathrm{~K}$, (b) annealed to $175 \mathrm{~K}$.

CS deposition. We prepared a base layer of crystalline $\mathrm{H}_{2} \mathrm{O}$ ice approximately $2.0 \mu \mathrm{m}$ thick by depositing $\mathrm{H}_{2} \mathrm{O}$ at $60 \mathrm{~K}$ and then annealing the ice to $175 \mathrm{~K}$. The ice was then cooled to $75 \mathrm{~K}$, and a layer of $\mathrm{CO}_{2}$ approximately $0.3 \mu \mathrm{m}$ thick was deposited on top of the water. The results of this layered ice can be seen in the top two spectra in Fig. 9. As these spectra clearly show, the features of crystalline $\mathrm{H}_{2} \mathrm{O}$ are still present and undistorted by the $\mathrm{CO}_{2}$ capping layer. Once the ice sample was heated above $110 \mathrm{~K}$, the $\mathrm{CO}_{2}$ completely desorbed.

The data from the CS deposition were also fit with the spectral components from pure water ices as discussed above and the fits are plotted along with the data in Fig. 9. In this case, the fits can reproduce qualitatively and quantitatively all the observed features at the selected temperatures, thus confirming that the $\mathrm{CO}_{2}$ is not affecting the structure of the crystalline $\mathrm{H}_{2} \mathrm{O}$ ice. The $6.8 \mathrm{THz}$ feature is, however, stronger in these CS data than in the fits. This difference is most likely because of the different experimental procedures used to prepared crystalline water ice.

AS deposition. We then repeated the experiment, but instead of crystalline $\mathrm{H}_{2} \mathrm{O}$, we deposited approximately $2.0 \mu \mathrm{m}$ of ASW at $60 \mathrm{~K}$ and then approximately $0.3 \mu \mathrm{m}$ of $\mathrm{CO}_{2}$ on top without changing the temperature. The THz spectra of this ice at $10 \mathrm{~K}$ and $60 \mathrm{~K}$ show the typical, broad feature from $4 \mathrm{THz}$ to $7.5 \mathrm{THz}$ of pure, amorphous $\mathrm{H}_{2} \mathrm{O}$. As the ice warms, however, the spectra of the layered sample deviate from the sample of the pure ice. In the blue trace in Fig. 9, we see the spectrum of this ice sample at $60 \mathrm{~K}$ after annealing to $100 \mathrm{~K}$. It appears that the broad amorphous band begins to change into the distinct bands of crystalline water, but that the process is not complete. However, when warmed to $150 \mathrm{~K}$, the crystalline $\mathrm{H}_{2} \mathrm{O}$ ice features become more apparent. Finally, when the ice is annealed to $175 \mathrm{~K}$ and then cooled back down to $150 \mathrm{~K}$, the ice features correspond to a well crystallized $\mathrm{H}_{2} \mathrm{O}$ ice which has been annealed. This can indeed be seen in both the THz spectra and in the mid-IR spectra presented in Fig. 9. In addition, spectra in the THz and mid-IR taken at $150 \mathrm{~K}$ show that the $\mathrm{CO}_{2}$ that was sitting on top of the $\mathrm{H}_{2} \mathrm{O}$ layer has completely left the ice.

There are, however, differences between the spectra of the CS deposition, seen in the top two spectra of Fig. 9, and the spectra of the AS deposition seen in the middle five spectra in Fig. 9. The CS deposition has a $\mathrm{THz}$ spectrum that appears qualitatively identical to the annealed pure ices in Fig. 7, while this is not the case for the AS experiments. The crystalline $\mathrm{H}_{2} \mathrm{O}$ features present in the AS spectra after annealing to $175 \mathrm{~K}$ are not as sharp or strong as the features present in both the pure annealed $\mathrm{H}_{2} \mathrm{O}$ ice or the ice in the CS deposition. From our quantitative fits of the data, we observe that up to $60 \mathrm{~K}$, the spectra can be fit with just an ASW component, while above $60 \mathrm{~K}$, a crystalline $\mathrm{H}_{2} \mathrm{O}$ at $150 \mathrm{~K}$ component must also be included to accurately fit the data. The percentage of the crystalline $\mathrm{H}_{2} \mathrm{O}$ component of the fit increases with increasing temperature as shown in Table 3, however since the total percentage does not approach $100 \%$, the structure of this ice, even after annealing, is somehow different from that of a pure crystalline $\mathrm{H}_{2} \mathrm{O}$ ice. Also, $\mathrm{CO}_{2}$ is still present in the ice at $100 \mathrm{~K}$ indicating that some $\mathrm{CO}_{2}$ diffused into the water layers underneath.

IS deposition. We then performed another experiment, where approximately $0.2 \mu \mathrm{m}$ of the more volatile $\mathrm{CO}_{2}$ was deposited directly onto the $\mathrm{Si}$ substrate at $60 \mathrm{~K}$, and then approximately $2.0 \mu \mathrm{m}$ of $\mathrm{H}_{2} \mathrm{O}$ was deposited as the top layer. These data can be seen in the bottom spectra of Fig. 9. Immediately after deposition, the sample was cooled to $10 \mathrm{~K}$ and spectra were collected. Above $100 \mathrm{~K}$, the ice was no longer stable because once enough $\mathrm{CO}_{2}$ begins to sublime from the ice, the $\mathrm{H}_{2} \mathrm{O}$ ice will no longer be in contact with the substrate and the ice will collapse. As such, these experiments were limited to the temperature range from $10 \mathrm{~K}$ to $100 \mathrm{~K}$.

As seen in Fig. 9 (right panel), both $\mathrm{CO}_{2}$ and $\mathrm{H}_{2} \mathrm{O}$ are present in the ice, and by looking at the shape of the $\mathrm{CO}_{2}$ bending mode peaks, we can tell that the ice layers remain mostly separate. The bending mode features show the three sharp peaks seen in pure $\mathrm{CO}_{2}$ as opposed to the one broadened feature seen in the well mixed ices discussed above. One would expect, then, to simply see $\mathrm{THz}$ features corresponding to amorphous $\mathrm{H}_{2} \mathrm{O}$; however, the $\mathrm{THz}$ spectrum is quite flat and nearly featureless. There does appear to be a subtle bump corresponding to the $4-7.5 \mathrm{THz}$ amorphous $\mathrm{H}_{2} \mathrm{O}$ ice absorption, but it is not especially intense. The $\mathrm{CO}_{2}$ molecules clearly disrupt the THz active motions in what should be an amorphous $\mathrm{H}_{2} \mathrm{O}$ even though amorphous $\mathrm{H}_{2} \mathrm{O}$ is clearly present from the FTIR data.

The layered ice was then warmed to $60 \mathrm{~K}$, and the $\mathrm{THz}$ features corresponding to ASW become more pronounced. While there is little change in the spectrum collected with the FTIR, it becomes possible to tell from the THz data that there is ASW present in the sample. The ice was then annealed to $100 \mathrm{~K}$ and cooled back to $60 \mathrm{~K}$. The spectrum after annealing now has 
a slightly different shape, but is qualitatively similar to the previous spectrum taken at $60 \mathrm{~K}$. While the mid-IR spectra do show some differences in this case, the differences are not as pronounced as in the $\mathrm{THz}$ region.

These spectra can be fit just with an ASW at $10 \mathrm{~K}$ component, except for the last spectrum annealed to $100 \mathrm{~K}$, where a crystalline $\mathrm{H}_{2} \mathrm{O}$ component at $150 \mathrm{~K}$ was included to improve the fit. In this case, we note that the proton-disordered vibrational band at 4.5 $\mathrm{THz}$ is present in the data and cannot be fit with the components hereby selected because the $6.8 \mathrm{THz}$ band is completely suppressed by the presence of the $\mathrm{CO}_{2}$ diffusing into the water top layers. A wider sample of spectral fitting components and a higher spectra resolution may explain the band at 4.5 THz.

Discussion of all three layered ices. Our results for the CS deposition agree well with literature results, ${ }^{36}$ as we observe all of the $\mathrm{CO}_{2}$ molecules desorb after heating the sample above $110 \mathrm{~K}$. We notice that the crystalline $\mathrm{H}_{2} \mathrm{O}$ spectra are in excellent agreement with the spectra of pure $\mathrm{H}_{2} \mathrm{O}$ presented in Fig. 7 .

When comparing the AS and CS experiments, the $\mathrm{THz}$ spectra provide more information about the structure of the ice when used in conjunction with the mid-IR data. The mid-IR spectra in Fig. 9 show that the water in the AS experiments crystallizes after annealing to $175 \mathrm{~K}$ and has significant similarities with the CS spectrum taken at $60 \mathrm{~K}$. However, the $\mathrm{THz}$ spectra of these two ices are quite different. As such, the $\mathrm{THz}$ spectra provide more information regarding the structure of the ices than just the mid-IR spectra do alone.

The AS deposition shows results that are different from the results obtained in the CS experiments, but that are similar to the results obtained from the different $\mathrm{CD}$ experiments reported above. These spectra are qualitatively similar to those in the CD experiment that was annealed directly after deposition, seen in the top half of Fig. 8. Both the spectra in Fig. 8 and 9 and show the 6.8, 5.7, and $4.7 \mathrm{THz}$ features of crystalline water ice that are attenuated and distorted relative to these features in pure crystalline $\mathrm{H}_{2} \mathrm{O}$ ice, and this can be seen in our fits. Clearly, the $\mathrm{CO}_{2}$ was able to diffuse into the structure of the ASW layer underneath and was trapped in the pores or channels of the ASW. ${ }^{37}$ The presence of these $\mathrm{CO}_{2}$ domains within the ASW prevented the crystallization process from proceeding as it would in the pure $\mathrm{H}_{2} \mathrm{O}$ ice. We can thus infer that, while there are large enough domains of water to crystallize, the presence of $\mathrm{CO}_{2}$ prevents these domains from joining to form a well ordered macroscopic crystal. Instead, crystallization is apparent in both the mid-IR and $\mathrm{THz}$ spectra, but the $\mathrm{THz}$ features are sensitive to the degree of crystallization of the ice and indicate that the ice differs in structure from that of a pure $\mathrm{H}_{2} \mathrm{O}$ sample.

The deposition in the IS experiment again points to the importance of $\mathrm{THz}$ spectroscopy in the study of the molecular structure of the ice. The fact that the feature corresponding to ASW is missing from these spectra is unsurprising from the results present in the literature. Maté et al. suggest that as $\mathrm{H}_{2} \mathrm{O}$ deposits, $\mathrm{CO}_{2}$ molecules are immediately incorporated into the structure of the ASW formed. ${ }^{37}$ This disruption of the structure of ASW would explain the flatness of the spectrum at $10 \mathrm{~K}$. Once the sample is heated to $60 \mathrm{~K}$, segregation begins to occur, and as Fig. 9 clearly shows, $\mathrm{CO}_{2}$ is still present in the ice after annealing to $100 \mathrm{~K}$. Consequently, a broad feature corresponding to that of ASW appears in both of the spectra taken at $60 \mathrm{~K}$ (before and after annealing to $100 \mathrm{~K}$ ). Since the mid-IR spectra sense only the local environment in which the molecules interact, the $\mathrm{THz}$ spectra provide the opportunity to draw stronger conclusions.

\section{Conclusions}

We present a new setup consisting of two spectrometers capable of studying astrochemical ice analogs from the $\mathrm{THz}$, or far-IR, region $\left(0.3-7.5 \mathrm{THz} ; 10-250 \mathrm{~cm}^{-1}\right)$ up through the mid-IR (400-4000 $\mathrm{cm}^{-1}$ ) with a $\mathrm{THz}$ spectral resolution of $3 \mathrm{~cm}^{-1}$ and a mid-IR spectral resolution of $1 \mathrm{~cm}^{-1}$. This combination of spectral regions is powerful because we are sensitive to intramolecular modes in the mid-IR and intermolecular modes in the THz. Also, we can compare our mid-IR spectrum to the large body of literature already available in this spectral region. In addition, the new setup described in this work is capable of making a significant contribution to the body of experimental laboratory data that is needed to interpret astronomical observations from Herschel, SOFIA, and ALMA. Below, we enumerate our conclusions:

1. Our $\mathrm{THz}$ spectra of pure $\mathrm{H}_{2} \mathrm{O}$ ice agree well with previous literature studies, and we tentatively report a new feature in both crystalline $\mathrm{H}_{2} \mathrm{O}$ and amorphous solid water at $1 \mathrm{THz}$. This feature may be especially useful for astronomical observations given the current coverage of observational facilities.

2. For experiments where we co-deposited $\mathrm{H}_{2} \mathrm{O}$ and $\mathrm{CO}_{2}$, we observed different structural changes within the $\mathrm{H}_{2} \mathrm{O}$ ice based on the rate of segregation. From the differences in the $\mathrm{THz}$ spectra, we observe that $\mathrm{CO}_{2}$ can segregate to disrupt the hydrogen bonding between bi-layers of crystalline $\mathrm{H}_{2} \mathrm{O}$ ice and thus infer the location of the segregated $\mathrm{CO}_{2}$.

3. In the experiments of sequentially deposited ices, we distinctly observed the impact of $\mathrm{CO}_{2}$ molecules on the structure of the $\mathrm{H}_{2} \mathrm{O}$ ice. Although we were unable to observe any features corresponding to pure $\mathrm{CO}_{2}$ ice, its effect on the $\mathrm{THz}$ spectrum was clear - $\mathrm{CO}_{2}$ disrupted the interactions between $\mathrm{H}_{2} \mathrm{O}$ molecules so that the resonances of $\mathrm{H}_{2} \mathrm{O}$ ice were attenuated, distorted, and/or shifted.

4. Given the differences in the $\mathrm{THz}$ spectra of the $\mathrm{CO}_{2}$ and $\mathrm{H}_{2} \mathrm{O}$ containing ices, $\mathrm{THz}$ spectroscopy has the potential to indicate the degree of crystallinity of an ice and the history of thermal transformations within the ice, provided that the history consists of non-equilibrium transitions away from a point of unstable equilibrium.

5. Our work demonstrates the importance of $\mathrm{THz}$ spectroscopy in the study of astrochemical ices because features in the $\mathrm{THz}$ region are sensitive to long range structural change. In addition, the temperature dependence of the shape of a feature in the THz spectrum of a molecule may provide a useful tool for determining the local temperature of an ice detected in space. 


\section{Acknowledgements}

The authors thank Daniel B. Holland and P. Brandon Carroll for helpful discussions as well as the referees whose comments helped improve the manuscript. This work was supported by the NSF CRIF:ID and CSDM programs and the NASA Exobiology and Laboratory Astrophysics programs. M.A.A thanks Katie Kirby for proofreading help, and was supported by the Department of Defense (DoD) Air Force Office of Scientific Research, National Defense Science and Engineering Graduate (NDSEG) Fellowship, 32 CFR 168a. S.I. acknowledges support from a Niels Stensen Fellowship and a Marie Curie Fellowship (FP7-PEOPLE-2011-IOF-300957). B.A.M. gratefully acknowledges funding by an NSF Graduate Research Fellowship.

\section{References}

1 R. Gould and E. Salpeter, Astrophys. J., 1963, 138, 393-407.

2 R. Gould, E. Salpeter and T. Gold, Astrophys. J., 1963, 138, 408.

3 S. Cazaux and A. G. G. M. Tielens, Astrophys. J., 2004, 604, 222.

4 H. B. Perets, O. Biham, G. Manic, V. Pirronello, J. Roser, S. Swords and G. Vidali, Astrophys. J., 2005, 627, 850.

5 L. Allamandola, M. Bernstein, S. Sandford and R. Walker, Space Sci. Rev., 1999, 90, 219-232.

6 E. F. van Dishoeck, Proc. Natl. Acad. Sci. U. S. A., 2006, 103, 12249-12256.

7 E. Herbst and E. F. van Dishoeck, Annu. Rev. Astron. Astrophys., 2009, 47, 427-480.

8 L. Allamandola, S. Sanford and G. Valero, Icarus, 1988, 76, 225-252.

9 M. Moore and R. Hudson, Icarus, 2000, 145, 282-288.

10 R. Hudson and M. Moore, Icarus, 2000, 145, 661-663.

11 G. Strazzulla, G. Baratta and M. Palumbo, Spectrochim. Acta, Part A, 2001, 57, 825-842.

12 S. Ioppolo, M. E. Palumbo, G. A. Baratta and V. Mennella, Astron. Astrophys., 2009, 493, 1017-1028.

13 U. Raut, D. Fulvio, M. J. Loeffler and R. A. Baragiola, Astrophys. J., 2012, 752, 159.

14 J. A. Noble, F. Dulieu, E. Congiu and H. J. Fraser, Astrophys. J., 2011, 735, 121.

15 P. Theule, F. Duvernay, A. Ilmane, T. Hasegawa, O. Morata, S. Coussan, G. Danger and T. Chiavassa, Astron. Astrophys., 2011, 530, A96.

16 J. A. Noble, P. Theule, F. Borget, G. Danger, M. Chomat, F. Duvernay, F. Mispelaer and T. Chiavassa, Mon. Not. R. Astron. Soc., 2013, 428, 3262-3273.

17 N. Watanabe and A. Kouchi, Astrophys. J., Lett., 2002, 571, L173.

18 G. W. Fuchs, H. M. Cuppen, S. Ioppolo, C. Romanzin, S. E. Bisschop, S. Andersson, E. F. van Dishoeck and H. Linnartz, Astron. Astrophys., 2009, 505, 629-639.

19 S. Ioppolo, H. M. Cuppen, C. Romanzin, E. F. van Dishoeck and H. Linnartz, Astrophys. J., 2008, 686, 1474.
20 N. Miyauchi, H. Hidaka, T. Chigai, A. Nagaoka, N. Watanabe and A. Kouchi, Chem. Phys. Lett., 2008, 456, 27-30.

21 E. Matar, E. Congiu, F. Dulieu, A. Momeni and J. L. Lemaire, Astron. Astrophys., 2008, 492, L17-L20.

22 U. Raut and R. A. Baragiola, Astrophys. J., Lett., 2011, 737, L14.

23 J. Kalvāns and I. Shmeld, Astron. Astrophys., 2010, 521, A37.

24 Q. Chang and E. Herbst, Astrophys. J., 2012, 759, 147.

25 R. L. Pulliam, B. A. McGuire and A. J. Remijan, Astrophys. J., 2012, 751, 1.

26 J. E. Elsila, D. P. Glavin and J. P. Dworkin, Meteorit. Planet. Sci., 2009, 44, 1323-1330.

27 R. T. Garrod, Astrophys. J., 2013, 765, 60.

28 W. Grundy, L. Young and E. Young, Icarus, 2003, 162, 222-229.

29 B. Buratti, D. Cruikshank, R. Brown, R. Clark, J. Bauer, R. Jaumann, T. McCord, D. Simonelli, C. Hibbitts, G. Hansen, T. Owen, K. Baines, G. Bellucci, J. Bibring, F. Capaccioni, P. Cerroni, A. Coradini, P. Drossart, V. Formisano, Y. Langevin, D. Matson, V. Mennella, R. Nelson, P. Nicholson, B. Sicardy, C. Sotin, T. Roush, K. Soderlund and A. Muradyan, Astrophys. J., 2005, 622, L149-L152.

30 R. T. Garrod, S. L. W. Weaver and E. Herbst, Astrophys. J., 2008, 682, 283-302.

31 J. R. Brucato, G. A. Baratta and G. Strazzulla, Astron. Astrophys., 2006, 455, 395-399.

32 S. E. Bisschop, G. W. Fuchs, A. C. A. Boogert, E. F. van Dishoeck and H. Linnartz, Astron. Astrophys., 2007, 470, 749-759.

33 K. I. Öberg, H. J. Fraser, A. C. A. Boogert, S. E. Bisschop, G. W. Fuchs, E. F. van Dishoeck and H. Linnartz, Astron. Astrophys., 2007, 462, 1187-1198.

34 K. I. Öberg, A. C. A. Boogert, K. M. Pontoppidan, S. van den Broek, E. F. van Dishoeck, S. Bottinelli, G. A. Blake and N. J. Evans, Astrophys. J., 2011, 740, 109.

35 M. Bernstein, D. Cruikshank and S. Sandford, Icarus, 2005, 179, 527-534.

36 O. Gálvez, I. K. Ortega, B. Maté, M. A. Moreno, B. MartnLlorente, V. J. Herrero, R. Escribano and P. J. Gutiérrez, Astron. Astrophys., 2007, 472, 691-698.

37 B. Maté, O. Galvez, B. Martin-Llorente, M. A. Moreno, V. J. Herrero, R. Escribano and E. Artacho, J. Phys. Chem. A, 2008, 112, 457-465.

38 O. Galvez, B. Maté, V. J. Herrero and R. Escribano, Icarus, 2008, 197, 599-605.

39 K. I. Öberg, E. C. Fayolle, H. M. Cuppen, E. F. van Dishoeck and H. Linnartz, Astron. Astrophys., 2009, 505, 183-194.

40 G. Profeta and S. Scandolo, Phys. Rev. B: Condens. Matter Mater. Phys., 2011, 84, 024103.

41 J. E. Bertie, Appl. Spectrosc., 1968, 22, 634-640.

42 J. E. Bertie and S. M. Jacobs, J. Chem. Phys., 1977, 67, 2445-2448.

43 M. Moore and R. Hudson, Astron. Astrophys., Suppl. Ser., 1994, 103, 45-56.

44 M. Moore and R. Hudson, Astrophys. J., 1992, 401, 353-360. 45 M. Moore and R. Hudson, Radiat. Phys. Chem., 1995, 45, 779-789. 
46 N. Plattner, M. W. Lee and M. Meuwly, Faraday Discuss., 2010, 147, 217-230.

47 M. W. Lee, N. Plattner and M. Meuwly, Phys. Chem. Chem. Phys., 2012, 14, 15464-15474.

48 J. D. Green, N. J. Evans II, J. K. Jorgensen, G. J. Herczeg, L. E. Kristensen, J.-E. Lee, O. Dionatos, U. A. Yildiz, C. Salyk, G. Meeus, J. Bouwman, R. Visser, E. A. Bergin, E. F. van Dishoeck, M. R. Rascati, A. Karska, T. A. van Kempen, M. M. Dunham, J. E. Lindberg, D. Fedele and the DIGIT Team, Astrophys. J., 2013, 770, 123.

49 R. Simon, N. Schneider, J. Stutzki, R. Guesten, U. U. Graf, P. Hartogh, X. Guan, J. G. Staguhn and D. J. Benford, Astron. Astrophys., 2012, 542, L12.

50 C. Qi, K. I. Öberg, D. J. Wilner, P. DAlessio, E. Bergin, S. M. Andrews, G. A. Blake, M. R. Hogerheijde and E. F. van Dishoeck, Science, 2013, 341, 630-632.

51 D. Cook and R. Hochstrasser, Opt. Lett., 2000, 25, 1210-1212.

52 X. Xie, J. Dai and X.-C. Zhang, Phys. Rev. Lett., 2006, 96, 075005.

53 K.-Y. Kim, J. H. Glownia, A. J. Taylor and G. Rodriguez, Opt. Express, 2007, 15, 4577-4584.

54 K. Y. Kim, A. J. Taylor, J. H. Glownia and G. Rodriguez, Nat. Photonics, 2008, 2, 605-609.

55 D. Auston, A. Johnson, P. Smith and J. Bean, Appl. Phys. Lett., 1980, 37, 371-373.
56 Q. Wu and X. Zhang, Appl. Phys. Lett., 1995, 67, 3523-3525. 57 P. A. Gerakines, W. A. Schutte, J. M. Greenberg and E. F. van Dishoeck, Astron. Astrophys., 1995, 296, 810.

58 M. S. Westley, G. A. Baratta and R. A. Baragiola, J. Chem. Phys., 1998, 108, 3321-3326.

59 M. Satorre, M. Domingo, C. Milln, R. Luna, R. Vilaplana and C. Santonja, Planet. Space Sci., 2008, 56, 1748-1752.

60 L. J. Karssenmeijer, S. Ioppolo, M. C. van Hermert, M. A. Allodi, G. A. Blake and H. M. Cuppen, Astrophys. J., in press.

61 J. B. Bossa, K. Isokoski, M. S. de Valois and H. Linnartz, Astron. Astrophys., 2012, 545, A82.

62 S. Krishnamurthy, R. Bansil and J. Wiafe-Akenten, J. Chem. Phys., 1983, 79, 5863-5870.

63 J. E. Bertie and E. Whalley, J. Chem. Phys., 1967, 46, 1271-1284.

64 D. D. Klug, J. S. Tse and E. Whalley, J. Chem. Phys., 1991, 95, 7011-7012.

65 D. M. Hudgins, S. A. Sandford, L. J. Allamandola and A. G. G. M. Tielens, Astrophys. J., Suppl. Ser., 1993, 86, 713-870.

66 P. Jenniskens and D. F. Blake, Astrophys. J., 1996, 473, 1104. 67 S. Ioppolo, G. Fedoseev, T. Lamberts, C. Romanzin and H. Linnartz, Rev. Sci. Instrum., 2013, 84, 073112.

68 E. C. Fayolle, K. I. Öberg, H. M. Cuppen, R. Visser and H. Linnartz, Astron. Astrophys., 2011, 529, A74. 